\title{
Tunable adhesion of a bio-inspired micro-pillar arrayed surface actuated by a magnetic field
}

\author{
Xingji Li ${ }^{\text {b,d }}$ Zhilong Peng ${ }^{\text {a, c }}$ Yazheng Yang ${ }^{\text {a, c }}$ Shaohua Chen ${ }^{\text {a, c,* }}$ \\ ${ }^{a}$ Institute of Advanced Structure Technology, Beijing Institute of Technology, Beijing, 100081, \\ China \\ ${ }^{\mathrm{b}}$ LNM, Institute of Mechanics, Chinese Academy of Sciences, Beijing, 100190, China \\ ${ }^{\mathrm{c}}$ Beijing Key Laboratory of Lightweight Multi-Functional Composite Materials and Structures, \\ Beijing Institute of Technology, Beijing, 100081, China \\ ${ }^{d}$ School of Engineering Science, University of Chinese Academy of Sciences, Beijing, 100049, \\ China
}

\section{Abstract}

Bio-inspired functional surfaces attract many research interests due to the promising applications. In this paper, tunable adhesion of a bio-inspired micro-pillar arrayed surface actuated by a magnetic field is investigated theoretically in order to disclose the mechanical mechanism of changeable adhesion and the influencing factors. Each PDMS micro-pillar reinforced by uniformly distributed magnetic-particles is assumed to be a cantilever beam. The beam's large elastic deformation is obtained under an externally magnetic field. Specially, the rotation angle of the pillar's end is predicted, which shows an essential effect on the changeable adhesion of the micro-pillar arrayed surface. The larger the strength of the applied magnetic field, the larger the rotation angle of the pillar's end will be, yielding a decreasing adhesion force of the micro-pillar arrayed surface. The difference of adhesion force tuned by the applied magnetic field can be a few orders of magnitude, which leads to controllable adhesion

\footnotetext{
*Corresponding author. Email address: chenshaohua72@hotmail.com or shchen@bit.edu.cn Chen 
of such a micro-pillar arrayed surface. Influences of each pillar's cross section shape, size, intervals between neighboring pillars and the distribution pattern on the adhesion force are further analyzed. The theoretical predictions are qualitatively well consistent with the experimental measurements. The present theoretical results should be helpful not only for the understanding of mechanical mechanism of tunable adhesion of micro-pillar arrayed surface under a magnetic field, but also for further precise and optimal design of such an adhesion-controllable bio-inspired surface in future practical applications.

Keywords: Micro-pillar arrayed surface; magnetic field; tunable adhesion; mechanical mechanism; large elastic deformation. 


\section{Introduction}

Many animals and insects possess strong and reversible adhesion on many surfaces, which has been attracting scientific research interests in order to disclose the mysterious mechanical mechanisms and fabricate bio-mimetically novel materials and devices [1-5]. Gecko, as a typical example, is capable of climbing on a vertical wall and even on a ceiling $[6,7]$. The mechanism underlying such a reversible adhesion behavior is mainly attributed to the elaborate hierarchical adhesion system $[4,5]$. It has been experimentally proved that the basic principle adopted by gecko is the van der Waals interaction $[6,7]$, while the capillary force $[8,9]$ and the electrostatic force [10] may also play significant roles.

In order to disclose the inherent mechanism of gecko adhesion and the influencing factors, many works have been carried out experimentally and theoretically [11-21]. The adhesive contact mechanics is often used to study the macro-adhesion mechanism of gecko. The oblique array and the hierarchical properties of gecko adhesion system were modeled as an anisotropic material [11], a graded material [12] and a hierarchical material [13-15]. It was found that the reversible adhesion could be achieved by changing the loading direction, which has been successfully proved by a bio-inspired experiment [16]. The graded and the hierarchical features are helpful for the strong adhesion [12-14]. In order to study the micro-adhesion mechanism, Gao et al. [13] modeled a gecko seta as a cylinder fiber, and found that saturation of the interfacial adhesion strength could be achieved as the radius of fiber is smaller than a 
critical size. Arzt et al. [4] found that splitting of a single contact into multiple smaller contacts can result in adhesion enhancement. Considering the real profile of gecko spatula, which is similar to a nano-thin film, the classical Kendall's peeling model [17] and the frictional adhesion model $[18,19]$ are often used to study the peeling behavior of a gecko spatula. It is found that the adhesion force is significantly influenced by the peeling angle, with which reversible adhesion between strong attachment and easy detachment can be achieved [18-20]. It has been widely accepted that gecko's reversible adhesion is realized mainly by the peeling behavior of its spatula pad [14, 21].

Inspired by geckos' adhesion system and adhesion mechanism, bio-inspired surfaces with micro- or nano-pillars have been successfully synthesized in order to study gecko adhesion in much more complex conditions, such as coupled temperature and humidity environment [22], underwater [23], and contaminated surface [24], by precisely adjusting the aspect ratio, tilting angle, contact shape and distribution patterns [25-28]. The adhesion strength of these bio-inspired synthetic surfaces could match and even pass the adhesive ability of geckos. However, how to realize reversible or tunable adhesion and how to fabricate spatula-like contact tip still need further investigations.

Fortunately, recent experiments found that micro-pillar arrayed surfaces could change the real contact area yielding tunable adhesion strength [28-30]. Two types of approaches are usually adopted to tune the surface contact area. The first way is 
through elastic large deformation of wedge-shape arrayed surfaces [31-33]. Through a loading-dragging-pulling (LDP) process, polymeric micro-wedges can be easily bent by the friction force, inducing a dramatically increasing contact area. Such a friction induced adhesion can be potentially used in micro-gravitational outer space for grasping and manipulating uncooperative objects [33]. Detachment can also be conveniently achieved by releasing the applied load to achieve the un-deformed state. Another way to change the contact area is via an external field. Drotlef et al. [34] have successfully prepared magnetic-particle contained PDMS micro-pillar arrayed surfaces, which would undergo significant bending deformation in response to an applied magnetic field. Thus, tunable adhesion could be realized by a varying magnetic field. Gillies et al. [35] studied the controllable particle adhesion with a magnetically actuated synthetic gecko adhesive, and also found that the adjustable adhesion could be achieved by the bending deformation of gecko-inspired micro-ridges under a magnetic field. The two methods to achieve tunable adhesion are well proposed [31-35]. However, in real applications, both precise design and accurate control are further required. How to achieve the industrialization in the future needs systematically theoretical analysis since some main factors cannot be systematically analyzed in the experiment due to the difficulty of sample preparation. What factors would influence the tunable adhesion still needs systematically theoretical analysis.

The present paper is mainly based on the well-done experiment by Drotlef et al. 
[34], in which micro-pillar arrayed surfaces with each micro-pillar filled with magnetic particles were fabricated. When an external magnetic field was applied, the micro-pillars on the surface would deform due to the magnetic force. Then, a spherical indenter was used to measure the adhesion force at each deformed configuration [34]. Only the van der Waals force between the spherical indenter and the deformed micro-pillar arrayed surface was mainly measured, similar to the geckos' adhesion mechanism. The van der Waals force between a single micro-pillar and the indenter is usually very small, but the whole adhesion force will be large enough due to the cumulative effect. It was found that the whole adhesion force of such a micro-pillar arrayed surface could be tuned by the external magnetic field.

In the present paper, a flat substrate is adopted to investigate the interaction force between deformed micro-pillar array and this flat substrate. First, the single micro-pillar is assumed to be a cantilever beam, whose elastic large bending deformation induced by an applied magnetic field is derived. Based on the bending configuration of the beam, closed-form solutions to adhesion strength of a single micro-pillar and a micro-pillar arrayed surface are further obtained. The effects of the micro-pillar cross section shape, size, intervals between neighboring pillars and the distribution pattern on the adhesion strength are analyzed in details. Comparison of the theoretical prediction and the experimental measurement is carried out finally. The results in the present paper should be helpful for further precise design of adhesion-tunable surfaces and devices. 


\section{Large bending deformation of a micro-pillar induced by a magnetic field}

According to the experimental sample [34], the schematic of a magnetic particle reinforced PDMS micro-pillar arrayed surface is shown in Fig. 1(a), in which the height and width of each pillar are denoted as $H$ and $a$, respectively. The interval between neighboring pillars is $b$. In Drotlef et al.'s experiment [34], the $\mathrm{NdFeB}$ micro-particles were provided by a commercial company. The shape and size of the particles cannot be absolutely identical. The $\mathrm{NdFeB}$ particles have platelet- or flake-like shape with an average size of $5 \mu \mathrm{m}$ [34]. For a theoretical model, even for a finite element model, the micro-particles in such a case are commonly assumed to be spherical ones with the same size without loss of generality.

In the experimental sample [34], all the magnetic particles in a micro-pillar are assumed to distribute continuously and uniformly in the height direction and be magnetized vertically. The externally applied magnetic field is exerted horizontally as shown in Fig. 1(a). The magnetic moment $\mathbf{p}_{m}$ that quantifies magnetization of one particle can be expressed as [36]

$$
\mathbf{p}_{m}=\frac{\mu_{r}-1}{\mu_{0} \mu_{r}} \nu \mathbf{B}_{R}
$$

where $\mu_{0}=4 \pi \times 10^{-7} \mathrm{~N} \cdot \mathrm{A}^{-2}$ is the permeability of vacuum, $\mu_{r}, v, \mathbf{B}_{R}$ are the relative permeability, volume and remanence of the particle, respectively. In the applied magnetic field, each magnetic particle gets a force moment, which can be 
expressed as the cross product of the magnetic moment $\mathbf{p}_{m}$ and the magnetic flux density B [34]

$$
\mathbf{M}=\mathbf{p}_{m} \times \mathbf{B}
$$

Each pillar is subjected to continuously distributed force moment and produces large bending deformation as shown in Fig. 1(b).

According to Drotlef et al.'s experiment [34], the aspect ratio of each micro-pillar is about 5, which can be reasonably assumed as an Euler-Bernoulli cantilever beam. Under the magnetic field, the tilting angle of each micro-pillar can reach to approximately $90^{\circ}$. Therefore, geometrical large-deformation theory should be used to analyze the bending deformation of the micro-pillar.

Considering large elastic deformation of the pillar, a curvilinear coordinate system $(s, \theta)$ and a rectangular coordinate system $(x, y)$ are introduced in Fig. 2(a) with the coordinate origin fixed at the bottom of the pillar, respectively. $s$ denotes the arc length of the neutral axis from the origin, $\theta$ is the rotation angle of each point on the neutral axis at $s$. The relation between the two coordinate systems is $\mathrm{d} x=\mathrm{d} s \cos \theta$ and $\mathrm{d} y=\mathrm{d} s \sin \theta$.

Substituting Eq. (1) into Eq. (2) yields $\mathbf{M}=\frac{\mu_{r}-1}{\mu_{0} \mu_{r}} v \mathbf{B}_{R} \times \mathbf{B}$. At the initial moment when the magnetic field is acted, the micro-pillar will keep a vertical configuration. The direction of the remanence of the particle $\mathbf{B}_{R}$ is perpendicular to the applied magnetic flux density $\mathbf{B}$. Thus, the force moment is a constant $M=\frac{\mu_{r}-1}{\mu_{0} \mu_{r}} V B_{R} B$, 
which distributes uniformly along the micro-pillar. When the bending deformation of the micro-pillar occurs, the angle between the two vectors $\mathbf{B}_{R}$ and $\mathbf{B}$ is $\pi / 2-\theta$ as shown in Fig. 1(c). In this case, the force moment becomes

$$
M(s, \theta)=\frac{\mu_{r}-1}{\mu_{0} \mu_{r}} V B_{R} B \cos \theta
$$

where $V$ is the particle volume per unit arc length. Due to the bending deformation, the rotation angle $\theta$ is a function of the arc length $s$. From Eq. (3), it is easy to find that the force moment $M$ varies with the arc length $s$ along the deformed micro-pillar.

The principle of minimum potential energy, as a basic method, is widely adopted to achieve the deformation of structures [37-39]. In the present paper, the principle of minimum potential energy is used to find the bending profile of each micro-pillar induced by the magnetic field.

The potential energy of a single micro-pillar can be written as

$$
\Pi=\frac{1}{2} \int_{0}^{H} \bar{E} I \theta^{\prime 2} \mathrm{~d} s-\int_{0}^{H} \mathrm{~d} s \int_{0}^{\theta} M(s, \theta) \mathrm{d} \theta+\int_{0}^{H} \lambda_{1}\left(x^{\prime}-\cos \theta\right) \mathrm{d} s+\int_{0}^{H} \lambda_{2}\left(y^{\prime}-\sin \theta\right) \mathrm{d} s
$$

where the first term on the right-hand side is the elastic bending energy, $\bar{E} I$ is the bending stiffness of the pillar. It should be noted that here $\bar{E}$ denotes the effective Young's modulus of the pillar, which is actually a particle reinforced polymer matrix composite. The second term is the potential of the force moment $M(s, \theta)$ induced by the magnetic field. $\lambda_{1}$ and $\lambda_{2}$ are two Lagrange multipliers enforcing the additionally geometrical conditions $\mathrm{d} x=\mathrm{d} s \cos \theta$ and $\mathrm{d} y=\mathrm{d} s \sin \theta$. 
The boundary conditions at the fixed end of the pillar can be expressed as

$$
\theta(0)=0, x(0)=0, y(0)=0
$$

The variation of Eq. (4) and the principle of minimum potential energy lead to

$$
\begin{aligned}
\delta \Pi & =\int_{0}^{H}\left[-\bar{E} I \theta^{\prime \prime}-M(s, \theta)+\lambda_{1} \sin \theta-\lambda_{2} \cos \theta\right] \delta \theta \mathrm{d} s-\int_{0}^{H} \lambda_{1}^{\prime} \delta x \mathrm{~d} s-\int_{0}^{H} \lambda_{2}^{\prime} \delta y \mathrm{~d} s \\
& +\int_{0}^{H}\left(x^{\prime}-\cos \theta\right) \delta \lambda_{1} \mathrm{~d} s+\int_{0}^{H}\left(y^{\prime}-\sin \theta\right) \delta \lambda_{2} \mathrm{~d} s \\
& +\left.\left(\bar{E} I \theta^{\prime} \delta \theta+\lambda_{1} \delta x+\lambda_{2} \delta y\right)\right|_{0} ^{H} \\
& =0
\end{aligned}
$$

Given the arbitrariness of the variation and considering the boundary conditions in Eq.

(5) yield

$$
\begin{gathered}
\bar{E} I \theta^{\prime \prime}+M(s, \theta)-\lambda_{1} \sin \theta+\lambda_{2} \cos \theta=0 \\
\lambda_{1}^{\prime}=0, \lambda_{2}^{\prime}=0 \\
x^{\prime}-\cos \theta=0, y^{\prime}-\sin \theta=0
\end{gathered}
$$

with additional boundary conditions

$$
\theta^{\prime}(H)=0, \lambda_{1}(H)=0, \lambda_{2}(H)=0
$$

Combining Eqs. (8) and (10) results in vanishing $\lambda_{1}$ and $\lambda_{2}$. The governing equation (7) and the boundary conditions in Eqs. (5) and (10) can be rewritten in a dimensionless form

$$
\begin{gathered}
\bar{\theta}^{\prime \prime}(\bar{s})+\bar{B} \cdot \cos \bar{\theta}(\bar{s})=0 \\
\bar{\theta}(0)=0, \bar{\theta}^{\prime}(1)=0
\end{gathered}
$$

where $\bar{B}(\bar{s}, \bar{\theta})=\frac{\left(\mu_{r}-1\right) B_{R} B}{\mu_{0} \mu_{r}} \frac{V H^{2}}{\bar{E} I}$ is the dimensionless magnetic field strength and $\bar{\theta}$ stands for the tangential angle with $\bar{s}=s / H$. Eq. (11) belongs to a typical 
boundary value problem of nonlinear ordinary differential equation, which can be solved numerically with a standard shoot method.

The deformed neutral axis of a micro-pillar induced by different applied magnetic fields is shown in Fig. 2(b), in which the deflection of the micro-pillar increases with increasing magnetic field strength.

\section{Adhesion force of a deformed micro-pillar under a magnetic field}

Fig. 3(a) shows a deformed micro-pillar arrayed surface interacting with an approaching flat substrate, which is different from the spherical indenter in the experiment [34].

In fact, in order to consider the adhesion problem of an elastic fiber or fiber arrays and a substrate, two kinds of model can be adopted. The first one is the contact model with a bilateral crack at the contact interface and an external load is assumed to pull or push two contact solids, which can be regarded as a model based on the continuum mechanics [13, 40-47]. In this model, the interface adhesive energy is introduced. Contact mechanics and fracture mechanics are used to find the interfacial stress field. Griffith fracture criterion is then adopted to find the relation between the external force and the contact size, from which the pull-off force of the adhesive interface can be achieved. Sometimes, the stress intensity factor is found from the fracture mechanics manual and the Griffith criterion is directly used to study the size effect of contact elements. Or the concept of interface flaw tolerance is adopted directly for a fiber in adhesive contact with a substrate, if the diameter of the fibers is less than a critical size. 
Then, the interfacial traction can be regarded as a uniformly distributed force and has nothing to do with the tip shape or deformation. The interface strength achieves the theoretical one. The second kind of model is mainly to find the interaction force between two approaching solids from the atomic interaction point of view. External load does not exist and only atom-atom interaction works between two approaching solids. Volume integral of L-J potential is usually used to find the whole interaction between the two approaching solids. In this kind of model, the effect of the deformation of the two approaching solids on the interaction force is often neglected due to the very small atomic lattice deformation and the difficulty of obtaining an exact solution under atom-atom interactions.

In this paper, we adopt the second kind of model to investigate the interaction between a deformed micro-pillar and a flat substrate, in which no external load exists and only the atom-atom interaction does. In addition, it is assumed that the magnetic force induced by the magnetic field is much larger than the van der Waals force acted on each micro-pillar, so that the deformation of a micro-pillar induced by the magnetic force is considered and the subsequent deformation of a bended micro-pillar induced by the van der Waals force is neglected.

With the above discussion and assumption, the adhesion force between two approaching solids can be achieved with the help of the following Lennard-Jones (L-J) potential [48] 


$$
w(r)=4 \varepsilon\left[\left(\frac{\sigma}{r}\right)^{12}-\left(\frac{\sigma}{r}\right)^{6}\right]
$$

where $\varepsilon$ is a parameter determining the depth of the potential well, $r$ is atom-to-atom distance, and $\sigma$ is a length scale parameter that determines the position of the zero potential.

The adhesion between a vertical pillar and a flat surface has been studied in [49] using the L-J potential method. For a bending pillar, the adhesion behavior is different from that of a vertical one because of different contacting surface morphologies. Furthermore, micro-pillars with flat tip but with different shaped cross sections will be considered in the present paper. According to the usually fabricated experimental samples, cases of square and circular cross sections are investigated. The bending deformation of the magnetic particle reinforced micro-pillar results from the applied magnetic field, which has been analyzed in section 2. Considering the effective interaction distance of van der Waals force, the interaction between the micro-pillar and the substrate is mainly contributed by the near-top volume of the micro-pillar. Contribution of the part far from the contact surface is relatively very weak. Therefore, for simplicity and without loss of generality, the bending micro-pillar can be treated as a tilting one as shown in Fig. 3(b) and Fig. 3(c), where the curvature effect of the bending micro-pillar is neglected, but the tilting angle $\varphi$ at the top of the tilting pillar takes the real rotation angle from the result of a deformed pillar under a magnetic field, i.e., $\varphi=\bar{\theta}(1)$. 


\subsection{Adhesion force of a deformed pillar of a square cross section}

The adhesion model between a pillar of a square cross section with a tilting angle $\varphi$ and a substrate is shown in Fig. 3(b), in which the space between the pillar and the substrate is $D$. The tilting angle $\varphi$ takes the real rotation value at the top of the magnetic particle reinforced pillar induced by an applied magnetic field.

After tedious derivation of the volume integration of L-J potential, the adhesion energy $W(D)$ between the tilting pillar and the substrate can be obtained as

$$
\begin{aligned}
W(D)= & \int_{V} w(r) \mathrm{d} V \\
= & \frac{4 \pi \varepsilon \rho_{1} \rho_{2} a}{\sin \varphi \cos \varphi}\left\{\left[\frac{1}{315}\left(1-\xi^{7}\right)-\frac{1}{360}\left(1-\xi^{8}\right)\right] \frac{\sigma^{12}}{D^{7}}-\left[\frac{1}{6}(1-\xi)-\frac{1}{12}\left(1-\xi^{2}\right)\right] \frac{\sigma^{6}}{D}\right\} \\
& +\frac{4 \pi \varepsilon \rho_{1} \rho_{2} a^{2}}{\cos \varphi}\left[\frac{1}{360}\left(\xi^{8}-\eta^{8}\right) \frac{\sigma^{12}}{D^{8}}-\frac{1}{12}\left(\xi^{2}-\eta^{2}\right) \frac{\sigma^{6}}{D^{2}}\right]
\end{aligned}
$$

where $\rho_{1}$ and $\rho_{2}$ denote the atomic densities of the pillar material and substrate material, respectively. $a$ is the side-length of the square cross section, and $\xi=D /(D+a \sin \varphi), \eta=D /(D+a \sin \varphi+H)$ are two dimensionless parameters.

It should be noted that, in the present paper, the magnetic particle reinforced micro-pillar is considered as a continuum material. An effective atomic density $\rho_{1}=v_{m} \rho_{m}+v_{p} \rho_{p}$ is adopted to denote the atomic density in the particle reinforced micro-pillar, where $v_{m}$ and $v_{p}$ are the volume fractions of the matrix and the particles in the composite material, $\rho_{m}$ and $\rho_{p}$ are the atomic densities of the pure matrix and particles, respectively.

The adhesion force between the deformed pillar of a square cross section and the 
substrate can then be obtained as

$$
F_{\text {ad }}=\frac{\partial W}{\partial D}=4 \pi \varepsilon \rho_{1} \rho_{2} a^{2}\left(-\alpha \frac{\sigma^{12}}{D^{9}}+\beta \frac{\sigma^{6}}{D^{3}}\right)
$$

where the two parameters are expressed as

$$
\begin{aligned}
& \alpha=\frac{1}{\cos \varphi}\left\{\frac{\xi}{1-\xi}\left[\frac{1}{40}\left(1-\xi^{8}\right)-\frac{1}{45}\left(1-\xi^{9}\right)\right]+\frac{1}{45}\left(\xi^{9}-\eta^{9}\right)\right\} \\
& \beta=\frac{1}{\cos \varphi}\left\{\frac{\xi}{1-\xi}\left[\frac{1}{4}\left(1-\xi^{2}\right)-\frac{1}{6}\left(1-\xi^{3}\right)\right]+\frac{1}{6}\left(\xi^{3}-\eta^{3}\right)\right\}
\end{aligned}
$$

\subsection{Adhesion force of a deformed pillar of a circular cross section}

The adhesion model between a pillar of a circular cross section with a tilting angle $\varphi$ and a substrate is shown in Fig. 3(c), where the diameter of the cross section of the pillar is $a . D$ denotes the distance between the pillar and the substrate. Similar to the square cross section case, the adhesion energy between the titling circular pillar and substrate can be achieved as

$$
W(D)=\frac{4 \pi^{2} \varepsilon \rho_{1} \rho_{2}}{\cos \varphi \sin ^{2} \varphi}\left\{\begin{array}{l}
{\left[\begin{array}{l}
\xi^{6}\left(\frac{33}{8} F(13)-\frac{63}{8} F(11)+\frac{35}{8} F(9)-\frac{5}{8} F(7)\right) \\
-\eta^{6}\left(\frac{33}{8} G(13)+\frac{63}{8} G(11)-\frac{35}{8} G(9)+\frac{5}{8} G(7)\right)
\end{array}\right] \frac{\sigma^{12}}{360 D^{6}}} \\
-[F(1)-G(1)] \frac{\sigma^{6}}{12}
\end{array}\right\}
$$

where $F(n)=\left[1-(1-\xi)^{2}\right]^{-n / 2}$ and $G(n)=\left[1-\eta^{2}(1-\xi)^{2} / \xi^{2}\right]^{-n / 2}$ with parameters $\xi=D /(D+a \sin \varphi / 2)$ and $\eta=D /(D+a \sin \varphi / 2+H)$.

The corresponding adhesion force can then be obtained as

$$
F_{\mathrm{ad}}=4 \pi \varepsilon \rho_{1} \rho_{2} \pi\left(\frac{a}{2}\right)^{2}\left(-\alpha \frac{\sigma^{12}}{D^{9}}+\beta \frac{\sigma^{6}}{D^{3}}\right)
$$


where the two parameters are

$$
\begin{aligned}
& \alpha=\frac{1}{45 \cos \varphi}\left(\frac{\xi}{1-\xi}\right)^{2}\left\{\begin{array}{l}
\xi^{7}\left[\frac{429}{64} F(15)-\frac{231}{16} F(13)+\frac{315}{32} F(11)-\frac{35}{16} F(9)+\frac{5}{64} F(7)\right] \\
-\eta^{7}\left[\frac{429}{64} G(15)-\frac{231}{16} G(13)+\frac{315}{32} G(11)-\frac{35}{16} G(9)+\frac{5}{64} G(7)\right]
\end{array}\right\} \\
& \beta=\frac{1}{6 \cos \varphi}\left(\frac{\xi}{1-\xi}\right)^{2}\{\xi[F(3)-F(1)]-\eta[G(3)-G(1)]\}
\end{aligned}
$$

\subsection{Adhesion force with a unified form}

Comparing Eqs. (14) and (17), we can write the adhesion force in a unified form for cases of pillars with different cross sections

$$
\bar{F}_{\text {ad }}=\frac{F_{\text {ad }}}{4 \pi \varepsilon \rho_{1} \rho_{2} \sigma^{3} H^{2}}=\bar{A}\left[-\alpha\left(\frac{\sigma}{D}\right)^{9}+\beta\left(\frac{\sigma}{D}\right)^{3}\right]
$$

where

$$
\bar{A}=\left\{\begin{array}{l}
\bar{a}^{2}, \text { for square pillar } \\
\frac{\pi \bar{a}^{2}}{4}, \text { for circular pillar }
\end{array}\right.
$$

and $\bar{a}=a / H$. Dimensionless parameters $\alpha$ and $\beta$ are expressed in Eqs. (15) for square pillars and in Eqs. (18) for circular pillars, respectively.

When the applied magnetic field vanishes, the micro-pillar will restore to the initially vertical configuration, i.e. $\varphi=0$ and we have $\xi=1$ and $\eta=D /(D+H)$. The unified adhesion force in Eq. (19) can be reduced to

$$
\bar{F}_{\mathrm{ad} 0}=\frac{F_{\mathrm{ad} 0}}{4 \pi \varepsilon \rho_{1} \rho_{2} \sigma^{3} H^{2}}=\bar{A}\left[-\frac{\sigma^{9}}{45 D^{9}}+\frac{\sigma^{3}}{6 D^{3}}+\frac{\sigma^{9}}{45(D+H)^{9}}-\frac{\sigma^{3}}{6(D+H)^{3}}\right]
$$

which is identical to the adhesion force between a flat punch and a substrate [49]. 


\section{Adhesion force of deformed micro-pillar arrays under a magnetic}

\section{field}

On bio-inspired micro-pillar arrayed surfaces, the micro-pillars are always designed to distribute in a regular pattern, such as, triangular, square and hexagonal patterns $[29,50,51]$. The adhesion behavior of a fibrillar array has been widely investigated with the contact mechanics and fracture mechanics method. Significant enhancement in adhesion of such a micro-pillar arrayed surface compared to that of a flat surface has been well explained from different points of view, for example, crack trapping, equal load sharing of each fibril, flaw tolerance $[43-45,51,52]$. In the present paper, the assumption of equal load sharing of each micro-pillar [44] is adopted due to the size of each pillar and the rigid substrate.

Fig. 4 gives three kinds of distribution patterns, i.e., triangular, square and hexagonal patterns for pillars of square and circular cross sections, respectively. Effects of the distribution pattern on the adhesion force will be analyzed. The area density $\phi$ of each kind of pillar array is defined as the ratio of each pillar's cross section area to the area encircled by a black dashed line as shown in Fig. 4. Thus, the area density $\phi$ can be expressed as $\phi=\phi_{\max } /(1+\rho)^{2}$ [51], where we define $\rho=b / a, a$ is the width or diameter of a pillar and $b$ is the interval of neighboring pillars. $\phi_{\max }$ is the maximum area density of a given pillar pattern when the closest neighboring upright pillars begin to contact each other. For the array consisting of pillars of square cross-section, we have $\phi_{\text {squ-3-max }}=\sqrt{3} / 2$ for a triangular pattern as 
shown in Fig. 4(a), $\phi_{\text {squ-4-max }}=1$ for a square pattern in Fig. $4(\mathrm{~b})$ and $\phi_{\text {squ-6-max }}=\sqrt{3} / 3$ for a hexagonal pattern in Fig. 4(c). It should be noted that not all pillars contact each other for the cases of $\phi_{\text {squ-3-max }}$ and $\phi_{\text {squ-6-max }}$ since the regular triangle and regular hexagonal patterns are assumed. For the array consisting of pillars of circular cross-section, the corresponding maximal area densities are $\phi_{\text {cir-3-max }}=\pi / 2 \sqrt{3}$, $\phi_{\text {cir-4-max }}=\pi / 4$ and $\phi_{\text {cir-6-max }}=\pi / 3 \sqrt{3}$, respectively, as shown in Fig. 4(d)-Fig. 4(f). In order to eliminate the effect of surface area, the adhesion force per unit area of distribution patterns is adopted,

$$
\bar{P}_{\mathrm{ad}}=\frac{\bar{F}_{\mathrm{ad}} \phi}{\bar{A}}=\frac{\bar{F}_{\mathrm{ad}} \phi_{\max }}{\bar{A}(1+\rho)^{2}}
$$

where $\bar{F}_{\text {ad }}$ and $\bar{A}$ are the adhesion force and the cross section area of a single pillar given in Eqs. (19) and (20), respectively.

\section{Results and discussion}

In the present paper, the homogenization method is adopted to find the equivalent mechanical property of particle reinforced composites, including the effective Young's modulus of composite materials $[53,54]$. The effect of ratio of the average particle diameter to the micro-pillar diameter or side is actually included by the volume fraction in the homogenization method.

Furthermore, in the experimental samples [34], surface roughness of each micro-pillar can not be avoided too. However, the aim of the present paper mainly focuses on the phenomenon of controllable adhesion force of the micro-pillar structure induced by the magnetic field. The scale of concern is the micro-pillar magnitude 
because the large deformation of each pillar is the main factor leading to the controllable adhesion force. A fine theoretical model considering the effect of micro-pillar surface roughness will be investigated in the future.

\subsection{The case of a micro-pillar under a magnetic field}

The tilting angle $\varphi$ at the top of the tilting pillar depends nonlinearly on the applied magnetic field $\bar{B}$, which can be found in Fig. 5. The stronger the magnetic field, the larger the tilting angle will be.

When a flat rigid substrate approaches the tilting micro-pillar, the adhesion force $\bar{F}_{\text {ad }}$ between the pillar and the substrate as given in Eq. (19) would be influenced not only by the applied magnetic field $\bar{B}$ but also by the distance $D / \sigma$ between the tilting pillar and the substrate. The adhesion force for both square and circular pillars with a given cross section area $\bar{A}=0.01$ under magnetic fields of different strengths is given in Fig. 6, where the adhesion force between the pillar and the substrate with $\bar{B}=0$ is also given for comparison. It is found that, for both square and circular pillars, the adhesion force between the pillar and the substrate varies with the separation distance in a van der Waals potential form. When the separation distance is relatively small, the interfacial interaction is repulsive. As the separation distance between the pillar and the substrate surpasses the equilibrium distance, at which the interfacial interaction vanishes, the interaction becomes attractive. Afterwards, the attractive force increases to a maximum and then drops quickly with the increasing separation distance. Finally, the interfacial interaction tends to vanish. When the separation 
distance is fixed, it is found that the adhesion force decreases with the increase of magnetic field strength. The effect of the cross section shape on the adhesion force is not very obvious only if the dimensionless cross section area $\bar{A}$ keeps the same. In the case of a vanishing applied magnetic field, the adhesion force of both the square and circular pillars are identical only if the dimensional cross section area remains the same as shown in Eq. (21).

Furthermore, Fig. 6 shows that the maximal adhesion force decreases when the applied magnetic field strength increases. Denote the maximal adhesion force as $\bar{F}_{\text {strength }}$, the effect of the magnetic field strength on the maximal adhesion force is depicted in Fig. 7 for both the square and the circular pillars of different cross section areas $\bar{A}$, in which the parameter $\bar{\sigma}=\sigma / H=3 \times 10^{-3}$ is adopted. It exhibits that the maximal adhesion force increases with the increase of the pillar's cross section for a fixed magnetic field strength. However, for a determined cross section area, the maximal adhesion force decreases drastically with the increasing applied magnetic field strength. Furthermore, the maximal adhesion force in all cases of different cross section areas would approach zero only if the applied magnetic field strength is large enough. All the results are mainly due to the effective interfacial interaction area, which varies with the variation of the applied magnetic field strength. Bending deformation of the micro-pillar would happen under an applied magnetic field, which subsequently induce the rotation of the pillar top. As a result, the real interacting area of the pillar with the substrate would decrease with the increase of the applied 
magnetic field strength. Such a magnetism-induced tunable contact configuration leads to tunable adhesion of a single pillar. Generally, the maximal adhesion force in the case of a circular cross section is a little bit smaller than that in the case of a square cross section when the dimensionless cross section area keeps the same.

\subsection{The case of a micro-pillar array under a magnetic field}

For a micro-pillar arrayed surface, the deformation of micro-pillar arrays will influence the adhesion ability of the surface since the adhesion force of the surface comes from the interaction between each pillar and the substrate. According to Eq. (22), many parameters would show influence on the adhesion force of a micro-pillar arrayed surface, such as the width or diameter of pillar $a$ and the interval $b$ between neighboring pillars (i.e. the value of $\rho=b / a$ ), the dimensionless cross section area $\bar{A}$, and the distribution pattern.

The maximal adhesion strength $\bar{P}_{\text {strength }}$ is introduced, which is defined as the maximal adhesion force per unit area, i.e.,

$$
\bar{P}_{\text {strength }}=\frac{\bar{F}_{\text {strength }} \phi}{\bar{A}}=\frac{\bar{F}_{\text {strength }} \phi_{\text {max }}}{\bar{A}(1+\rho)^{2}}
$$

Here, one can see that the maximal adhesion strength is a function of $\bar{A}, \rho$ (i.e., $\bar{a}$ or $\bar{b}), \phi_{\max }$ and the maximal adhesion force.

For a micro-pillar with a square or circular cross section, once the width or diameter of the pillar $\bar{a}$ is given, the cross section area $\bar{A}$ is determined. Then, the value of $\rho=\bar{b} / \bar{a}$ for a micro-pillar arrayed surface in any kind of pattern depends only on the interval $\bar{b}$. Since the maximal adhesion force of the surface is influenced Chen 
by the applied magnetic field strength, the relation between the maximal adhesion strength and the applied magnetic field strength can be achieved for cases of different intervals as shown in Fig. 8(a) for a square pillar arrayed surface in a square pattern and Fig. 8(b) for the circular one in a square pattern, respectively, where the parameters are $\bar{a}=0.1$ and $\bar{\sigma}=\sigma / H=3 \times 10^{-3}$. It is obvious that the maximal adhesion strength decreases with the increase of the applied magnetic field strength in the case of a certain interval $\bar{b}$. Furthermore, the maximal adhesion strength is reduced by the increase of the interval of neighboring pillars in the case of a determined applied magnetic field strength. Such a result should be very easily understood since the larger the applied magnetic field strength, the larger the rotation angle of each pillar's top is; and the larger the interval of neighboring pillars, the smaller the area density of the pillar arrays is. As a result, the maximal adhesion force would be reduced by the increase of the applied magnetic field strength or the interval between neighboring pillars.

If the interval between neighboring pillars is fixed, the effect of the pillar's width or diameter $\bar{a}$ on the maximal adhesion strength of the micro-pillar arrayed surface in a square pattern can be obtained. Figs. 9(a) and 9(b) show the relation between the maximal adhesion strength and the applied magnetic field strength for the square pillar arrayed surface case and the circular one, respectively, where $\bar{b}=0.1$ and $\bar{\sigma}=\sigma / H=3 \times 10^{-3}$. It is found that the maximal adhesion strength increases with the increase of the pillar's width or diameter, especially for the case with a relatively weak 
applied magnetic field strength. It means that the adhesion strength is proportional to the area density of pillar arrays. However, the maximal adhesion strength becomes insensitive to the pillar's width or diameter when the applied magnetic field strength is relatively large. It is due to the large deformation of pillars and the large rotation angle at pillar tips, both of which are induced by a strong applied magnetic field strength.

All the above effects of the width or diameter of pillars and the interval of neighboring pillars on the maximal adhesion strength are also true for micro-pillar arrayed surfaces in other distribution patterns.

The effect of distribution pattern of micro-pillar arrayed surfaces on the maximal adhesion strength is further investigated. Three kinds of distribution patterns as shown in Fig. 4, i.e., triangular, square and hexagonal ones, are taken into consideration for both square and circular micro-pillar arrayed surfaces. The relation between the maximal adhesion strength and the applied magnetic field strength is given in Fig. 10 for cases of different distribution patterns. All the parameters adopted in Fig. 10 are $\rho=1$ with $\bar{a}=\bar{b}=0.1$ and $\bar{\sigma}=\sigma / H=3 \times 10^{-3}$. It is found that no matter what the distribution pattern of micro-pillar array is and what shape of each pillar's cross section is, the maximal adhesion strength always decreases with the increase of the applied magnetic field strength. However, the distribution pattern in cases of micro-pillars of different cross sections will show influence on the maximal adhesion strength of the micro-pillar arrayed surfaces, especially under a relatively weak applied magnetic field strength. For a square pillar arrayed surface, the maximal 
adhesion strength in the case of square distribution pattern is much larger than that in the case of triangular or hexagonal distribution pattern. While for a circular pillar arrayed surface, the maximal adhesion strength in the case of triangular distribution pattern is much larger than that in the case of square or hexagonal distribution pattern. Among all the combining cases, the maximal adhesion strength of a square pillar arrayed surface in a square pattern achieves the maximum, while the maximal adhesion strength of a square pillar arrayed surface in a hexagonal pattern is the smallest. It is interesting to find that the setal mat in geckos' foot is also arranged in a tetrad pattern [23], which is well consistent with the present results. When the applied magnetic field strength is relatively strong, difference of the effects of the distribution pattern is not very obvious. The maximal adhesion in all the cases tends to vanish.

Comparing the maximal adhesion strength under different applied magnetic field strength finds that there is an order of magnitude difference. It means that tunable adhesion could be realized on such a bio-inspired micro-pillar arrayed surface by the applied magnetic field. All the micro-pillars on the surface are vertical under a vanishing external magnetic field, at which the maximal adhesion strength achieves the maximum. While all the micro-pillars bend seriously and the maximal adhesion strength approaches to zero when the applied magnetic field strength is large enough. The adhesion of such a magnetic particle reinforced micro-pillar arrayed surface can be well tuned by the applied magnetic field.

In order to attain good tunable adhesion performance, one can design a square 
patterned surface consisting of pillars of a square cross section due to the largest area density if the width and interval are determined. The circular pillar arrayed surface in a triangular pattern might be also popular due to the second largest area density and the easily preparation technology $[29,50]$.

From the theoretical analysis, it is found that the way to enlarge the pillar's width and to reduce the interval between neighboring pillars at the same time can increase the upper limit of the maximal adhesion strength. However, the pillar's width and the interval cannot be adjusted randomly, which should avoid the adhesion between the neighboring pillars due to the van der Waals interaction. Adhesion of neighboring pillars may lead to cluster and prevent deformed pillars from restoring the initial configuration in the absence of magnetic field. Such a problem has been studied by Gao et al. [13] and Glassmaker et al. [42], respectively. Another issue in the present investigated magnetic particle reinforced micro-pillar arrayed surface is that bending deformation of neighboring pillars would happen in the same direction when an external magnetic field is added. If the interval of neighboring micro-pillars decreases, one of the pillar's edge would contact another one. Let $\varphi_{\mathrm{cr}}$ denote the critical tilting angle, at which two neighboring pillars contact each other due to the bending deformation. Then, the contact condition as shown in Fig. 1(b) can be expressed as $a+b=a / \cos \varphi_{\mathrm{cr}}$. Considering $\rho=b / a$ yields the critical ratio $\rho_{\mathrm{cr}}=1 / \cos \varphi_{\mathrm{cr}}-1$.

The relationship between the critical ratio $\rho_{\text {cr }}$ and the applied magnetic field strength $\bar{B}$ is further shown in Fig. 11, in which the critical curve divides the plane 
into two regions: one is the no bundling (no contact) region of the upper left and another is the bundling (contact) region of the lower right. With the increase of the magnetic field strength $\bar{B}$, a relatively large interval $b$ is needed and the width or diameter of the pillar $a$ can not be arbitrarily large in order to avoid neighboring contact, which apparently contradicts with the condition of achieving strong and tunable adhesion. In fact, it gives a limiting condition on all the above results and discussion. Therefore, in practical fabrications and applications, comprehensive design of the ratio $\rho$ is needed. It should be noted that all the values of $\rho$ taken in the present work are valid in the no bundling region.

\subsection{Comparison of the theoretical result and the experimental measurement}

Corresponding to the present theoretical model, similar experiments have been well done by Drotlef et al. [34], in which the effect of magnetic field on the adheison of NdFeB micro-particle reinforced PDMS micro-pillar arrayed surfaces was investigated. Under an applied magnetic field, large bending deformation of the micro pillars was observed via microscopy. The adhesion force varying with the tilting angle of the pillars was measured with the help of adhesion tester with a spherical probe. It was found that the adhesion force decreased with an increasing tilting angle induced by the applied magnetic field (please see Fig. 3C in ref. [34]). Comparison of the experimental measurement and the present theoretical prediction as given in Fig. 12 shows good qualitative agreement.

It should be noted that quantitative comparison between the present theoretical 
model and the experimental result can not be carried out due to several aspects: the first one is the adhesion force in experiment was measured between the micro-pillar arrayed surface and a spherical probe, while a flat substrate is adopted for simplicity in the present theoretical model; as a result the real interaction area between a micro-pillar arrayed surface and a spherical probe is difficult to obtain. The second one is some material parameters in experiments were not given, for example, the effecive Young' modulus of the pillar, the magnetic flux density, the bending stiffness of the pillar, etc. Besides the van der Waals force, the electrostatic and capillary interactions between the micro-pillar array and substrate are hardly to avoid in experiment [55], which is not considered in the present theoretical model. However, similar mechanism underlying the magnetic field actuated tunable adhesion of a micro-pillar arrayed surface found in experiment can be reasonably predicted in the present theoretical analysis and qualitative prediction of the adhesion force and adhesion strength influenced by several factors in the theoretical model should be helpful for further precise designs of reversible or tunable adhesive sensors.

\section{Conclusions}

Inspired by tunable adhesion of a magnetic particle reinforced PDMS micro-pillar arrayed surface actuated by an applied magnetic field, we establish theoretical models to analyze the mechanical mechanism of tunable adhesion and the main influencing factors of adhesion. Large deformation of each pillar is considered and surfaces consisting of micro-pillars of square and circular cross sections are investigated. The 
adhesion mechanism of a single pillar is studied first. The tunable adhesion is mainly due to the varying rotation angle of the pillar's tip, which results from the large deformation of pillars induced by the applied magnetic field. Further analysis of micro-pillars of different cross sections distributing in different patterns is carried out. It is found that under a fixed magnetic field, the adhesion between a micro-pillar arrayed surface and a substrate varies with the separation distance in a van der Waals interaction form, in which a maximum adhesion strength exists. The maximum adhesion strength in each case decreases with the increase of the applied magnetic field strength, leading to tunable adhesion. Analysis of the influence of pillar's cross section shapes, the intervals between neighboring pillars and the distribution patterns exhibits that the surface consisting of square pillars distributing in a square pattern would possess the best tunable adhesion feature if the cross section area is fixed. All the theoretical results agree well with the existing experimental measurements qualitatively [34] and should be helpful for precise design of bio-inspired functional surfaces of reversible or tunable adhesion in the future.

\section{Acknowledgements}

The work reported here is supported by NSFC through Grants \#11532013, \#11672302, \#11872114 and the BIT Creative Research Plan. 


\section{References}

[1] Gorb, S., and Scherge, M., 2000, "Biological Microtribology: Anisotropy in Frictional Forces of Orthopteran Attachment Pads Reflects the Ultrastructure of a Highly Deformable Material," Proc. R. Soc. B, 267(1449), pp. 1239-1244.

[2] Federle, W., Brainerd, E. L., McMahon, T. A., and Holldobler, B., 2001, "Biomechanics of the Movable Pretarsal Adhesive Organ in Ants and Bees," Proc. Natl. Acad. Sci. USA, 98(11), pp. 6215-6220.

[3] Federle, W., Baumgartner, W., and Holldobler, B., 2004, "Biomechanics of Ant Adhesive Pads: Frictional Forces Are Rate- and Temperature-Dependent," J. Exp. Biol., 207(1), pp. 67-74.

[4] Arzt, E., Gorb, S., and Spolenak, R., 2003, "From Micro to Nano Contacts in Biological Attachment Devices," Proc. Natl. Acad. Sci. USA, 100(19), pp. 10603-10606.

[5] Autumn, K., and Gravish, N., 2008, "Gecko Adhesion: Evolutionary Nanotechnology," Philos. Trans. R. Soc. A, 366(1870), pp. 1575-1590.

[6] Autumn, K., Liang, Y. A., Hsieh, S. T., Zesch, W., Chan, W. P., Kenny, T. W., Fearing, R., and Full, R. J., 2000, "Adhesive Force of a Single Gecko Foot-Hair," Nature, 405(6787), pp. 681-685.

[7] Autumn, K., Sitti, M., Liang, Y. A., Peattie, A. M., Hansen, W. R., Sponberg, S., Kenny, T. W., Fearing, R., Israelachvili, J. N., and Full, R. J., 2002, "Evidence for Van Der Waals Adhesion in Gecko Setae," Proc. Natl. Acad. Sci. USA, 99(19), pp. $12252-12256$. 
[8] Huber, G., Mantz, H., Spolenak, R., Mecke, K., Jacobs, K., Gorb, S. N., and Arzt, E., 2005, "Evidence for Capillarity Contributions to Gecko Adhesion from Single Spatula Nanomechanical Measurements," Proc. Natl. Acad. Sci. USA, 102(45), pp. 16293-16296.

[9] Sun, W. X., Neuzil, P., Kustandi, T. S., Oh, S., and Samper, V. D., 2005, "The Nature of the Gecko Lizard Adhesive Force," Biophys. J., 89(2), pp. L14-L17.

[10] Izadi, H., Stewart, K. M., and Penlidis, A., 2014, "Role of Contact Electrification and Electrostatic Interactions in Gecko Adhesion," J. R. Soc. Interface, 11(98), p. 20140371.

[11] Chen, S. H., and Gao, H. J., 2007, "Bio-Inspired Mechanics of Reversible Adhesion: Orientation-Dependent Adhesion Strength for Non-Slipping Adhesive Contact with Transversely Isotropic Elastic Materials," J. Mech. Phys. Solids, 55(5), pp. 1001-1015.

[12] Chen, S. H., Yan, C., Zhang, P., and Gao, H. J., 2009, "Mechanics of Adhesive Contact on a Power-Law Graded Elastic Half-Space," J. Mech. Phys. Solids, 57(9), pp. $1437-1448$.

[13] Gao, H. J., Wang, X., Yao, H. M., Gorb, S., and Arzt, E., 2005, "Mechanics of Hierarchical Adhesion Structures of Geckos," Mech. Mater., 37(2-3), pp. 275-285.

[14] Chen, B., Wu, P. D., and Gao, H. J., 2008, "Hierarchical Modelling of Attachment and Detachment Mechanisms of Gecko Toe Adhesion," Proc. R. Soc. A, 464(2094), pp. $1639-1652$.

[15] Peng, Z. L., and Chen, S. H., 2012, "Effect of Pre-Tension on the Peeling Behavior 
of a Bio-Inspired Nano-Film and a Hierarchical Adhesive Structure," Appl. Phys. Lett., 101(16), p. 163702.

[16] Lee, J. H., Fearing, R. S., and Komvopoulos, K., 2008, "Directional Adhesion of Gecko-Inspired Angled Microfiber Arrays," Appl. Phys. Lett., 93(19), p. 191910.

[17] Kendall, K., 1975, "Thin-Film Peeling - Elastic Term," J. Phys. D Appl. Phys., 8(13), pp. 1449-1452.

[18] Autumn, K., Dittmore, A., Santos, D., Spenko, M., and Cutkosky, M., 2006, "Frictional Adhesion: A New Angle on Gecko Attachment," J. Exp. Biol., 209(Pt 18), pp. 3569-3579.

[19] Tian, Y., Pesika, N., Zeng, H. B., Rosenberg, K., Zhao, B. X., McGuiggan, P., Autumn, K., and Israelachvili, J., 2006, "Adhesion and Friction in Gecko Toe Attachment and Detachment," Proc. Natl. Acad. Sci. USA, 103(51), pp. 19320-19325. [20] Peng, Z. L., Chen, S. H., and Soh, A. K., 2010, "Peeling Behavior of a Bio-Inspired Nano-Film on a Substrate," Int. J. Solids Struct., 47(14-15), pp. 1952-1960.

[21] Chen, B., Wu, P. D., and Gao, H. J., 2009, "Pre-Tension Generates Strongly Reversible Adhesion of a Spatula Pad on Substrate," J. R. Soc. Interface, 6(35), pp. $529-537$.

[22] Stark, A. Y., Klittich, M. R., Sitti, M., Niewiarowski, P. H., and Dhinojwala, A., 2016, "The Effect of Temperature and Humidity on Adhesion of a Gecko-Inspired Adhesive: Implications for the Natural System," Sci. Rep., 6, p. 30936.

[23] Stark, A. Y., Badge, I., Wucinich, N. A., Sullivan, T. W., Niewiarowski, P. H., and Dhinojwala, A., 2013, "Surface Wettability Plays a Significant Role in Gecko Adhesion 
Underwater," Proc. Natl. Acad. Sci. USA, 110(16), pp. 6340-6345.

[24] Xu, Q., Wan, Y. Y., Hu, T. S., Liu, T. X., Tao, D. S., Niewiarowski, P. H., Tian, Y., Liu, Y., Dai, L. M., Yang, Y. Q., and Xia, Z. H., 2015, "Robust Self-Cleaning and Micromanipulation Capabilities of Gecko Spatulae and Their Bio-Mimics," Nat. Commun., 6, p. 8949.

[25] Micciche, M., Arzt, E., and Kroner, E., 2014, "Single Macroscopic Pillars as Model System for Bioinspired Adhesives: Influence of Tip Dimension, Aspect Ratio, and Tilt Angle," ACS Appl. Mater. Interfaces, 6(10), pp. 7076-7083.

[26] Carbone, G., and Pierro, E., 2013, "A Review of Adhesion Mechanisms of Mushroom-Shaped Microstructured Adhesives," Meccanica, 48(8), pp. 1819-1833.

[27] Aksak, B., Sahin, K., and Sitti, M., 2014, "The Optimal Shape of Elastomer Mushroom-Like Fibers for High and Robust Adhesion," Beilstein. J. Nanotech., 5, pp. 830-838.

[28] Varenberg, M., Murarash, B., Kligerman, Y., and Gorb, S. N., 2011, "Geometry-Controlled Adhesion: Revisiting the Contact Splitting Hypothesis," Appl. Phys. A Mater. Sci. Process., 103(4), pp. 933-938.

[29] Hu, H., Tian, H. M., Shao, J. Y., Wang, Y., Li, X. M., Tian, Y., Ding, Y. C., and Lu, B. H., 2017, "Friction Contribution to Bioinspired Mushroom-Shaped Dry Adhesives," Adv. Mater. Interfaces, 4(9), p. 1700016.

[30] Murphy, M. P., Kim, S., and Sitti, M., 2009, "Enhanced Adhesion by Gecko-Inspired Hierarchical Fibrillar Adhesives," ACS Appl. Mater. Interfaces, 1(4), pp. 849-855. 
[31] Tao, D. S., Gao, X., Lu, H. Y., Liu, Z. Y., Li, Y., Tong, H., Pesika, N., Meng, Y. G., and Tian, Y., 2017, "Controllable Anisotropic Dry Adhesion in Vacuum: Gecko Inspired Wedged Surface Fabricated with Ultraprecision Diamond Cutting," Adv. Funct. Mater., 27(22), p. 1606576.

[32] Wu, X. A., Christensen, D. L., Suresh, S. A., Jiang, H., Roderick, W. R., and Cutkosky, M., 2017, "Incipient Slip Detection and Recovery for Controllable Gecko-Inspired Adhesion," IEEE Robot. Autom. Lett., 2(2), pp. 460-467.

[33] Jiang, H., Hawkes, E. W., Fuller, C., Estrada, M. A., Suresh, S. A., Abcouwer, N., Han, A. K., Wang, S., Ploch, C. J., Parness, A., and Cutkosky, M. R., 2017, "A Robotic Device Using Gecko-Inspired Adhesives Can Grasp and Manipulate Large Objects in Microgravity," Sci. Robot., 2(7), p. eaan4545.

[34] Drotlef, D. M., Blumler, P., and del Campo, A., 2014, "Magnetically Actuated Patterns for Bioinspired Reversible Adhesion (Dry and Wet)," Adv. Mater., 26(5), pp. 775-779.

[35] Gillies, A. G., Kwak, J., and Fearing, R. S., 2013, "Controllable Particle Adhesion with a Magnetically Actuated Synthetic Gecko Adhesive," Adv. Funct. Mater., 23(26), pp. 3256-3261.

[36] Feynman, R. P., Leighton, R. B., and Sands, M. L., 1965, The Feynman Lectures on Physics: Vol. 2: The Electromagnetic Field, Addison-Wesley.

[37] Peng, Z. L., and Chen, S. H., 2015, "Effect of Bending Stiffness on the Peeling Behavior of an Elastic Thin Film on a Rigid Substrate," Phys. Rev. E, 91(4), p. 042401. [38] Tang, T., Jagota, A., and Hui, C. Y., 2005, "Adhesion between Single-Walled 
Carbon Nanotubes," J. Appl. Phys., 97(7).

[39] Zhang, C., Chen, L., and Chen, S. H., 2013, "Adhesion between Two Radially Collapsed Single-Walled Carbon Nanotubes," Acta Mech., 224(11), pp. 2759-2770.

[40] Johnson, K. L., Kendall, K., and Roberts, A. D., 1971, "Surface Energy and the Contact of Elastic Solids," Proc. R. Soc. Lond. A, 324(1558), pp. 301-313.

[41] Gao, H. J., and Yao, H. M., 2004, "Shape Insensitive Optimal Adhesion of Nanoscale Fibrillar Structures," Proc. Natl. Acad. Sci. USA, 101(21), pp. 7851-7856.

[42] Glassmaker, N. J., Jagota, A., Hui, C. Y., and Kim, J., 2004, "Design of

Biomimetic Fibrillar Interfaces: 1. Making Contact," J. R. Soc. Interface, 1(1), pp. 23-33.

[43] Glassmaker, N. J., Jagota, A., Hui, C. Y., Noderer, W. L., and Chaudhury, M. K., 2007, "Biologically Inspired Crack Trapping for Enhanced Adhesion," Proc. Natl. Acad. Sci. USA, 104(26), pp. 10786-10791.

[44] Hui, C. Y., Glassmaker, N. J., Tang, T., and Jagota, A., 2004, "Design of Biomimetic Fibrillar Interfaces: 2. Mechanics of Enhanced Adhesion," J. R. Soc. Interface, 1(1), pp. 35-48.

[45] Jagota, A., and Bennison, S. J., 2002, "Mechanics of Adhesion through a Fibrillar Microstructure," Integr. Comp. Biol., 42(6), pp. 1140-1145.

[46] Guo, X., Jin, F., and Gao, H. J., 2011, "Mechanics of Non-Slipping Adhesive Contact on a Power-Law Graded Elastic Half-Space," Int. J. Solids Struct., 48(18), pp. 2565-2575.

[47] Jin, F., Guo, X., and Gao, H. J., 2013, "Adhesive Contact on Power-Law Graded 
Elastic Solids: The JKR-DMT Transition Using a Double-Hertz Model," J. Mech. Phys. Solids, 61(12), pp. 2473-2492.

[48] Jones, J. E., 1924, "On the Determination of Molecular Fields - II from the Equation of State of a Gas," Proc. R. Soc. London A, 106(738), pp. 463-477.

[49] Israelachvili, J. N., 2011, Intermolecular and Surface Forces: Revised Third Edition, Academic Press.

[50] Greiner, C., del Campo, A., and Arzt, E., 2007, "Adhesion of Bioinspired Micropatterned Surfaces: Effects of Pillar Radius, Aspect Ratio, and Preload," Langmuir, 23(7), pp. 3495-3502.

[51] Yao, H. M., and Gao, H. J., 2006, "Mechanics of Robust and Releasable Adhesion in Biology: Bottom-up Designed Hierarchical Structures of Gecko," J. Mech. Phys. Solids, 54(6), pp. 1120-1146.

[52] Gorb, S., Varenberg, M., Peressadko, A., and Tuma, J., 2007, "Biomimetic Mushroom-Shaped Fibrillar Adhesive Microstructure," J. R. Soc. Interface, 4(13), pp. 271-275.

[53] Liang, J. Z., 2013, "Reinforcement and Quantitative Description of Inorganic Particulate-Filled Polymer Composites," Compos. Part B Eng., 51, pp. 224-232.

[54] Turcsányi, B., Pukánszky, B., and Tüdõs, F., 1988, "Composition Dependence of Tensile Yield Stress in Filled Polymers," J. Mater. Sci. Lett., 7(2), pp. 160-162.

[55] Komvopoulos, K., and Yan, W., 1997, "A Fractal Analysis of Stiction in Microelectromechanical Systems," J. Tribol., 119(3), pp. 391-400. 


\section{Figure captions}

Fig. 1 Schematics of the theoretical model. (a) The model of a magnetic particle reinforced PDMS micro-pillar arrayed surface, in which the height and width of each pillar are $H$ and $a . b$ denotes the interval of neighboring pillars. The micro-pillars keep vertical without an applied magnetic field. (b) Under an applied magnetic field $\mathbf{B}$, large bending deformation happens for micro-pillars. $\varphi$ is the rotation angle at the pillar's top. (c) The relation of vectors of the remanence $\mathbf{B}_{R}$, the magnetic field $\mathbf{B}$ and the moment $\mathbf{M}$ acting on a single particle. Here, $\theta$ is the rotation angle of the pillar where the particle locates.

Fig. 2 Schematics of large deformed cantilever beam model. (a) A rectangular coordinate system $(x, y)$ and a curvilinear coordinate system $(s, \theta)$ are attached on the initial and deformed configurations, respectively. (b) The profiles of a deformed cantilever beam under different magnetic field strength.

Fig. 3 Schematics of the interaction between a micro-pillar arrayed surface and a substrate. (a) Under an applied magnetic field, the deformed micro-pillars are approached by a substrate, where $D$ is the separation distance and $\varphi$ is the rotation angle at the pillar's top. (b) A tilting square shaped micro-pillar interacts with a substrate, where $a$ is the width of the square cross section. (c) A tilting circular micro-pillar interacts with a substrate, where $a$ is the diameter of the circular cross section.

Fig. 4 Top views of micro-pillar's distribution patterns. (a)-(c) Triangular, square and 
hexagonal distribution patterns for square pillars; (d)-(f) Triangular, square and hexagonal distribution patterns for circular pillars. The area surrounded by dashed lines denotes one cell of the pattern. The maximal area density in each pattern is given at the bottom of each figure.

Fig. 5 The relation between the rotation angle of the pillar's top $\varphi$ and the applied magnetic field strength $\bar{B}$.

Fig. 6 The dimensionless adhesion force $\bar{F}_{\text {ad }}$ between a single tilting pillar and a substrate as a function of the separation distance $D / \sigma$ under different magnetic field strength, where the cross section area is taken as $\bar{A}=0.01$ for both square and circular pillars. $\left(\bar{\sigma}=\sigma / H=3 \times 10^{-3}\right)$

Fig. 7 The dimensionless maximal adhesion strength $\bar{F}_{\text {strength }}$ varying with the applied magnetic field $\bar{B}$ for both square and circular cross section cases with the cross section area of $\bar{A}=0.01,0.0225$ and 0.04 , respectively. $\left(\bar{\sigma}=\sigma / H=3 \times 10^{-3}\right)$

Fig. 8 The dimensionless maximal adhesion strength $\bar{P}_{\text {strength }}$ as a function of the applied magnetic field strength $\bar{B}$ for micro-pillar arrays distributed in a square pattern but with different intervals $\bar{b}$ between neighboring pillars. (a) For the case of square cross section. (b) For the case of circular cross section. In both cases, $\bar{a}=a / H=0.1$ and $\bar{\sigma}=\sigma / H=3 \times 10^{-3}$.

Fig. 9 The dimensionless maximal adhesion strength $\bar{P}_{\text {strength }}$ as a function of the applied magnetic field strength $\bar{B}$ for micro-pillar arrays distributed in a square pattern but with pillars of different widths or diameters $\bar{a}$. (a) For the 
case of square cross section. (b) For the case of circular cross section. In both cases, $\bar{b}=b / H=0.1$ and $\bar{\sigma}=\sigma / H=3 \times 10^{-3}$.

Fig. 10 The dimensionless maximal adhesion strength $\bar{P}_{\text {strength }}$ varying with the applied magnetic field strength $\bar{B}$ for both square and circular micro-pillar arrays but with different distribution patterns, where $\rho=1$ with $\bar{a}=\bar{b}=0.1$ and $\bar{\sigma}=\sigma / H=3 \times 10^{-3}$.

Fig. 11 The critical ratio $\rho_{\text {cr }}$ as a function of the applied magnetic field strength $\bar{B}$, which can divide the plane into two regions.

Fig. 12 The maximal adhesion strength $\bar{P}_{\text {strength }}$ varying with the rotation angle of pillar's top $\varphi$ for a arrayed surface with circular pillars arranged in square pattern, in which $\bar{a}=\bar{b}=0.1$ and $\bar{\sigma}=\sigma / H=3 \times 10^{-3}$. 

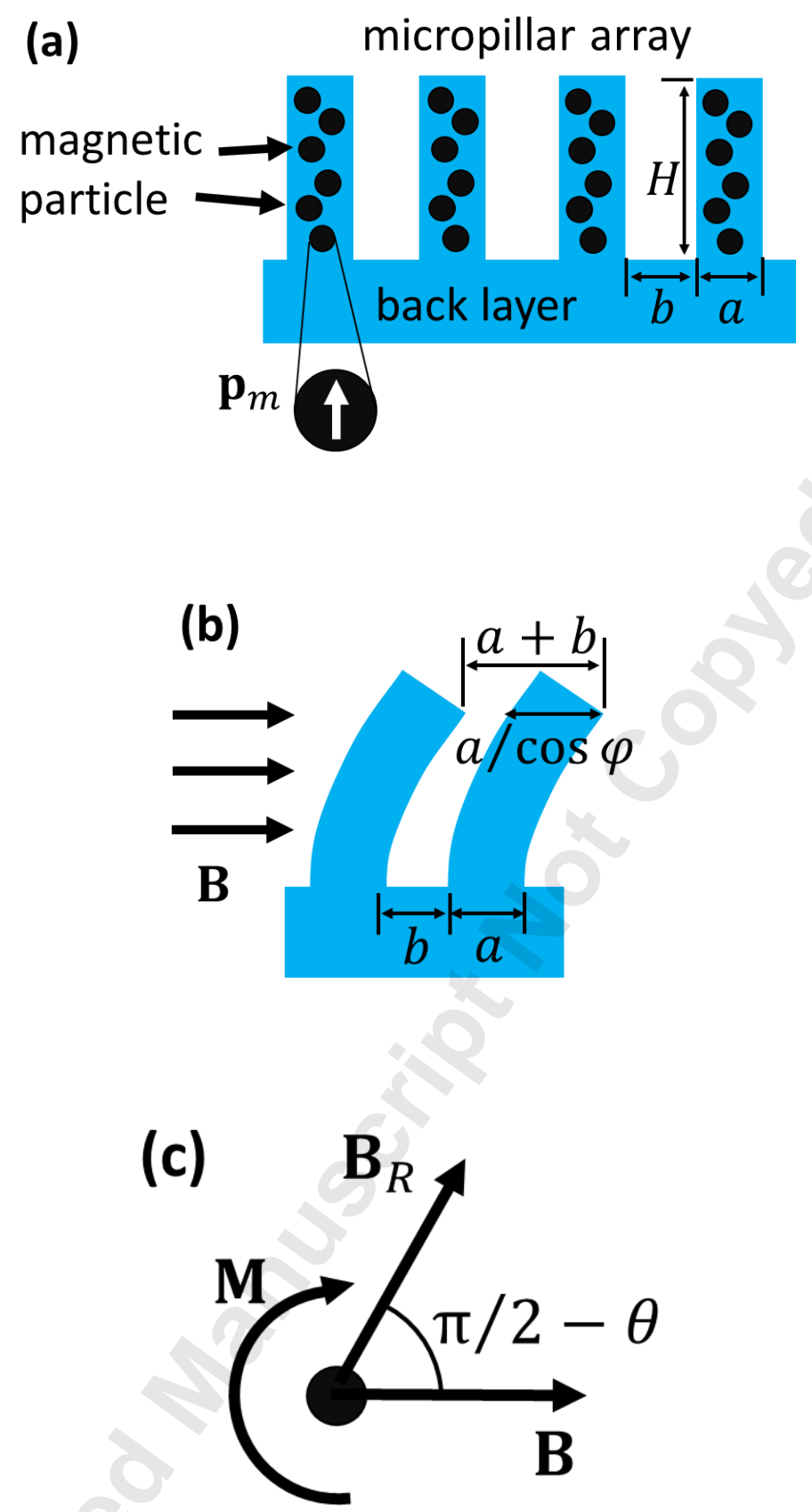

Fig. 1 

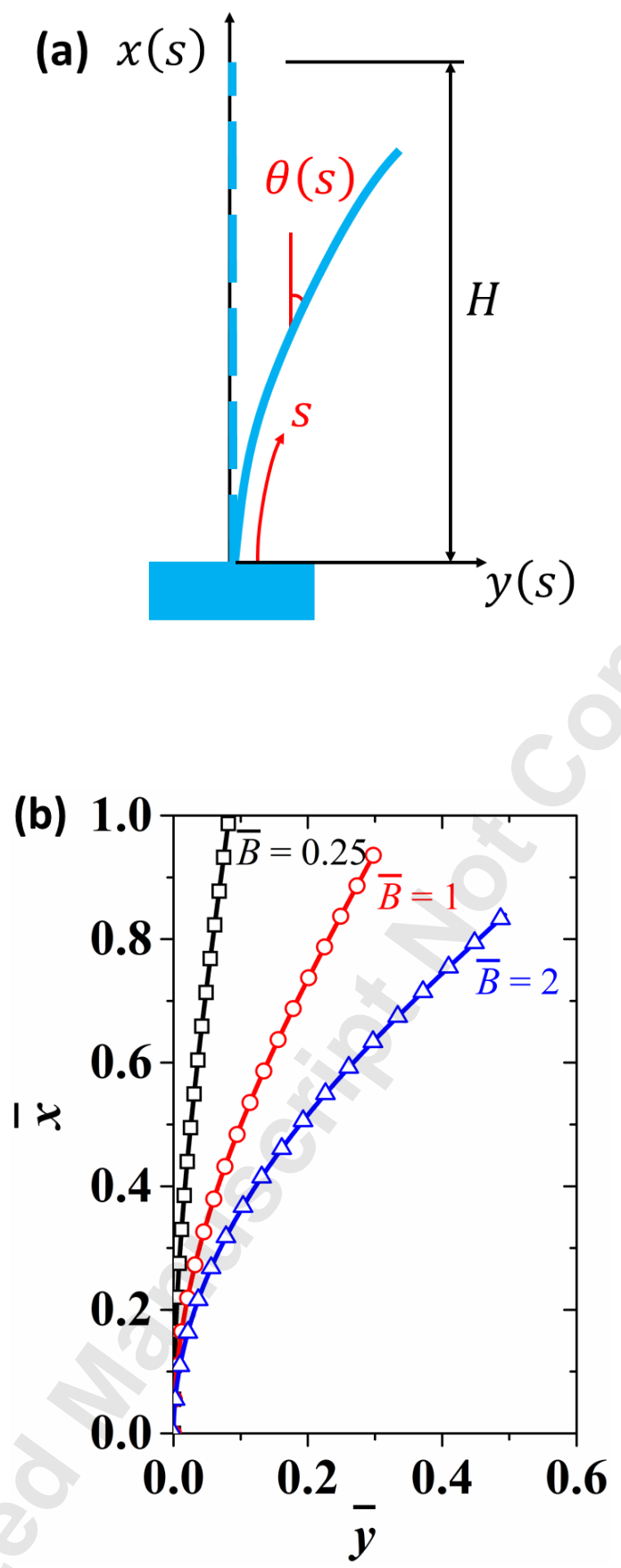

Fig. 2 


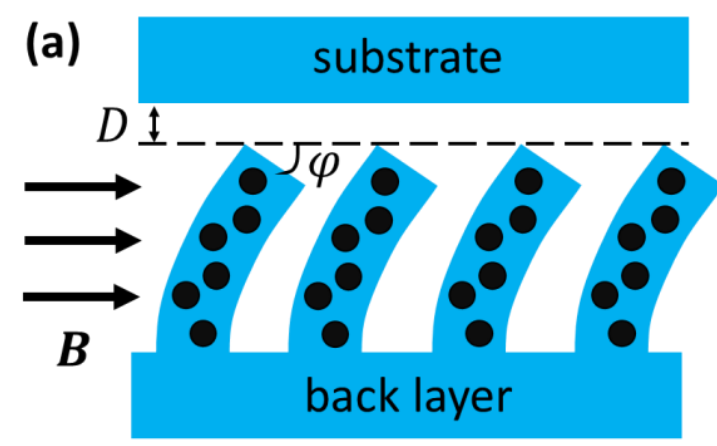

(b)

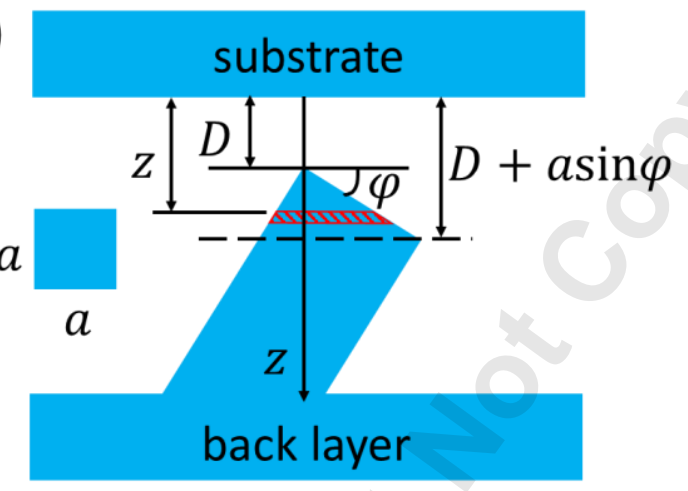

(c)

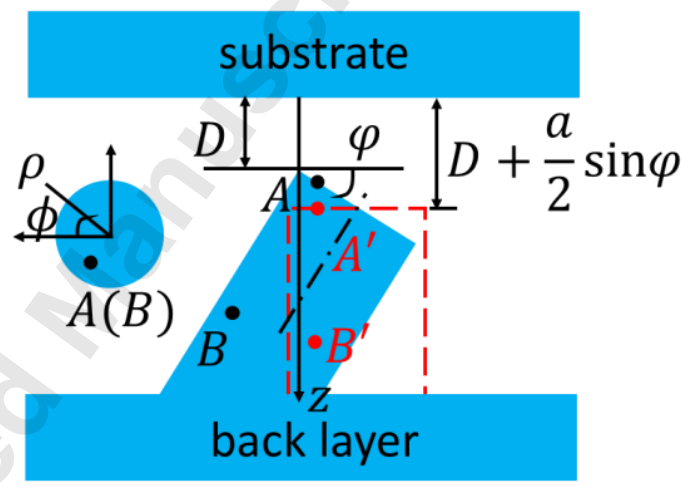

Fig. 3 

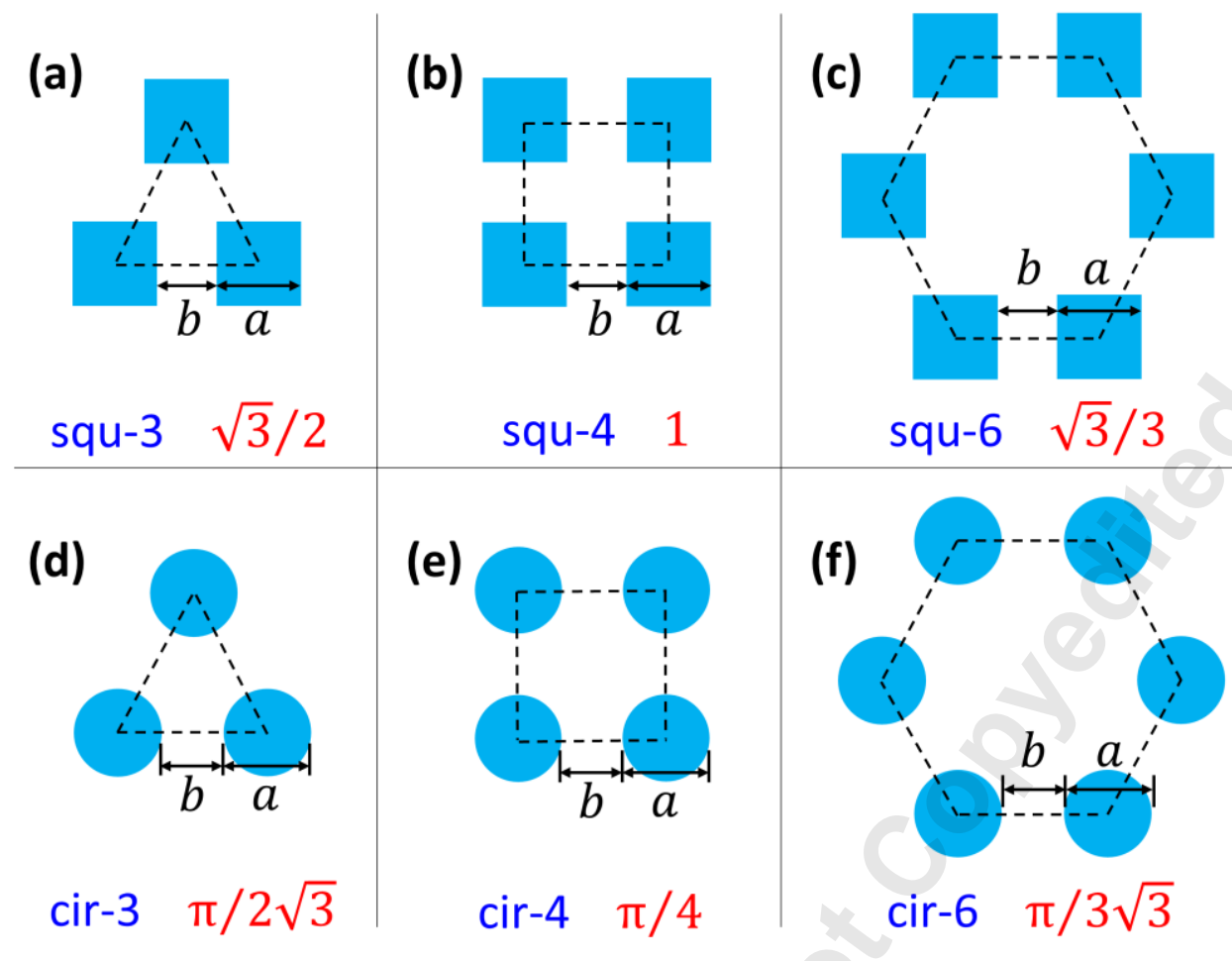

squ-6 $\sqrt{3} / 3$

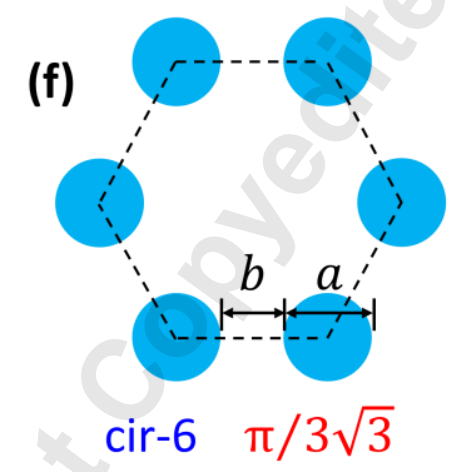

Fig. 4 


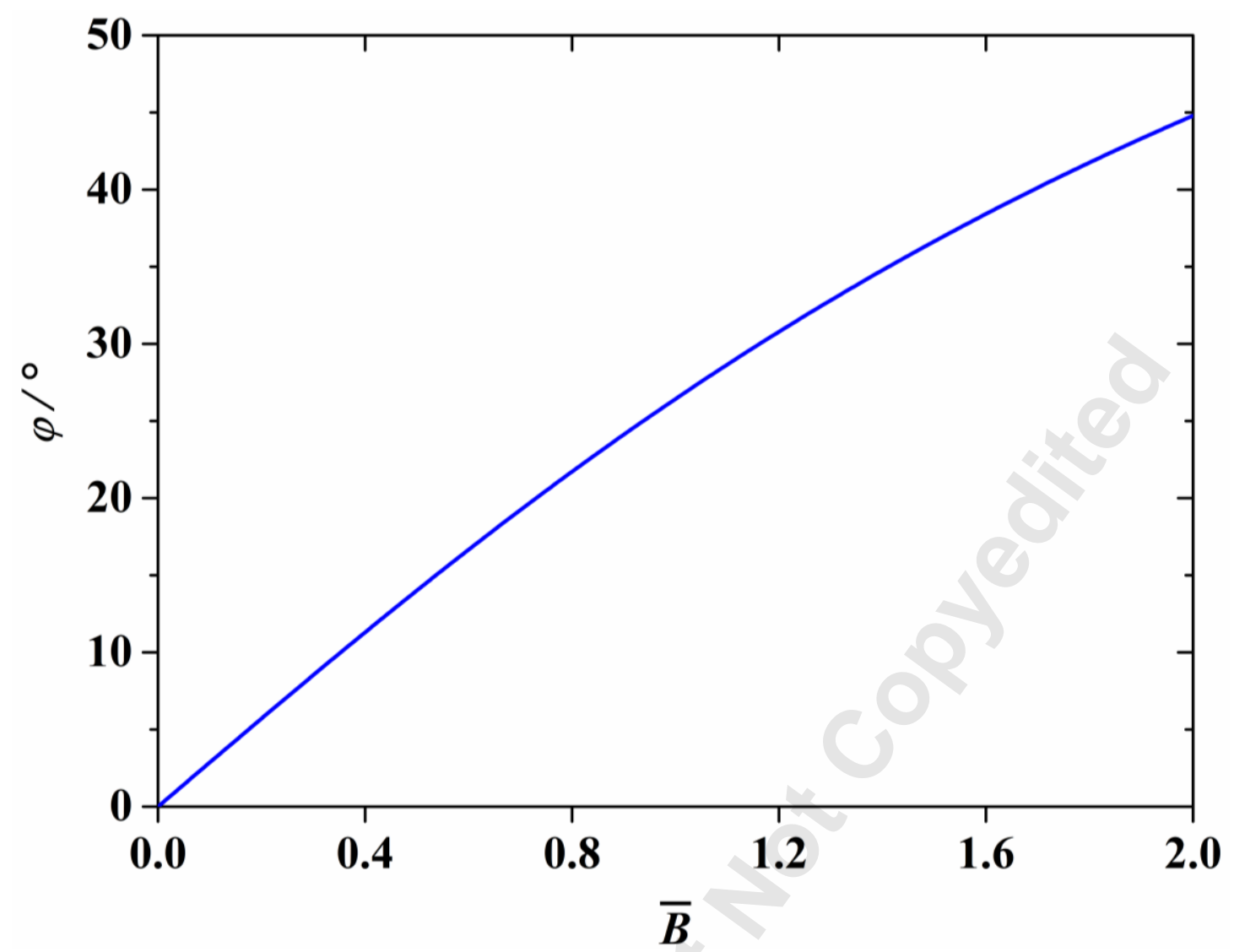

Fig. 5 


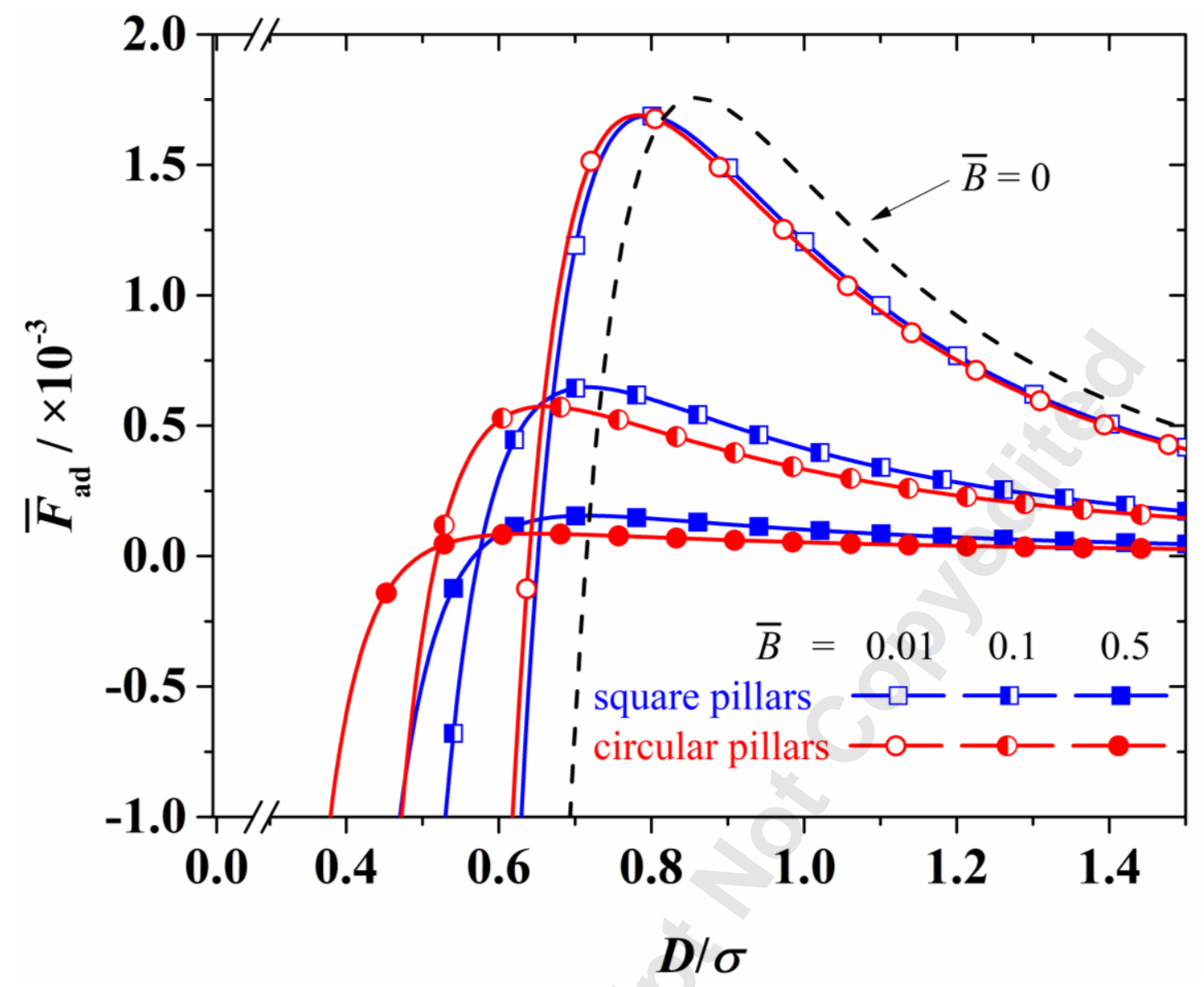

Fig. 6 


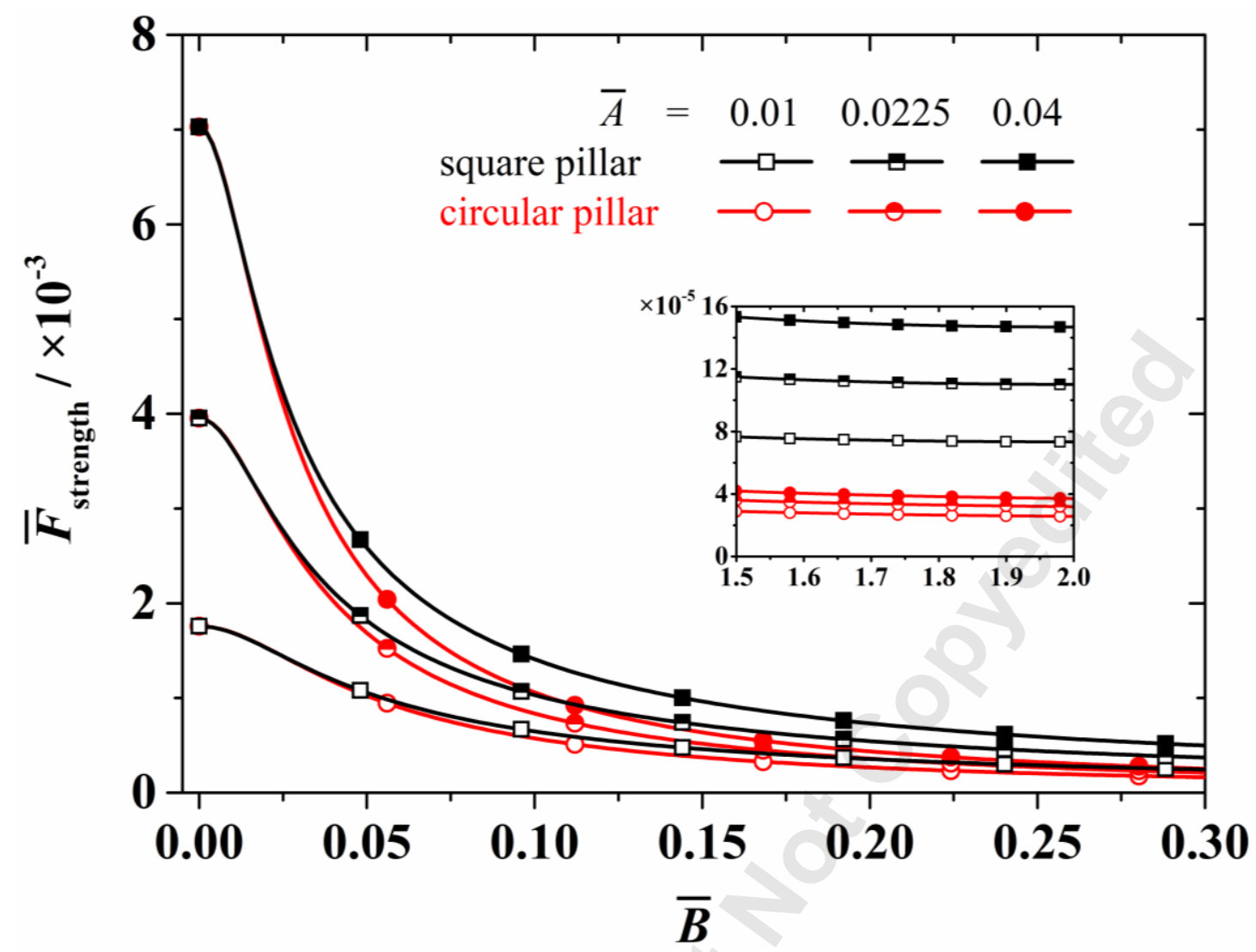

Fig. 7 

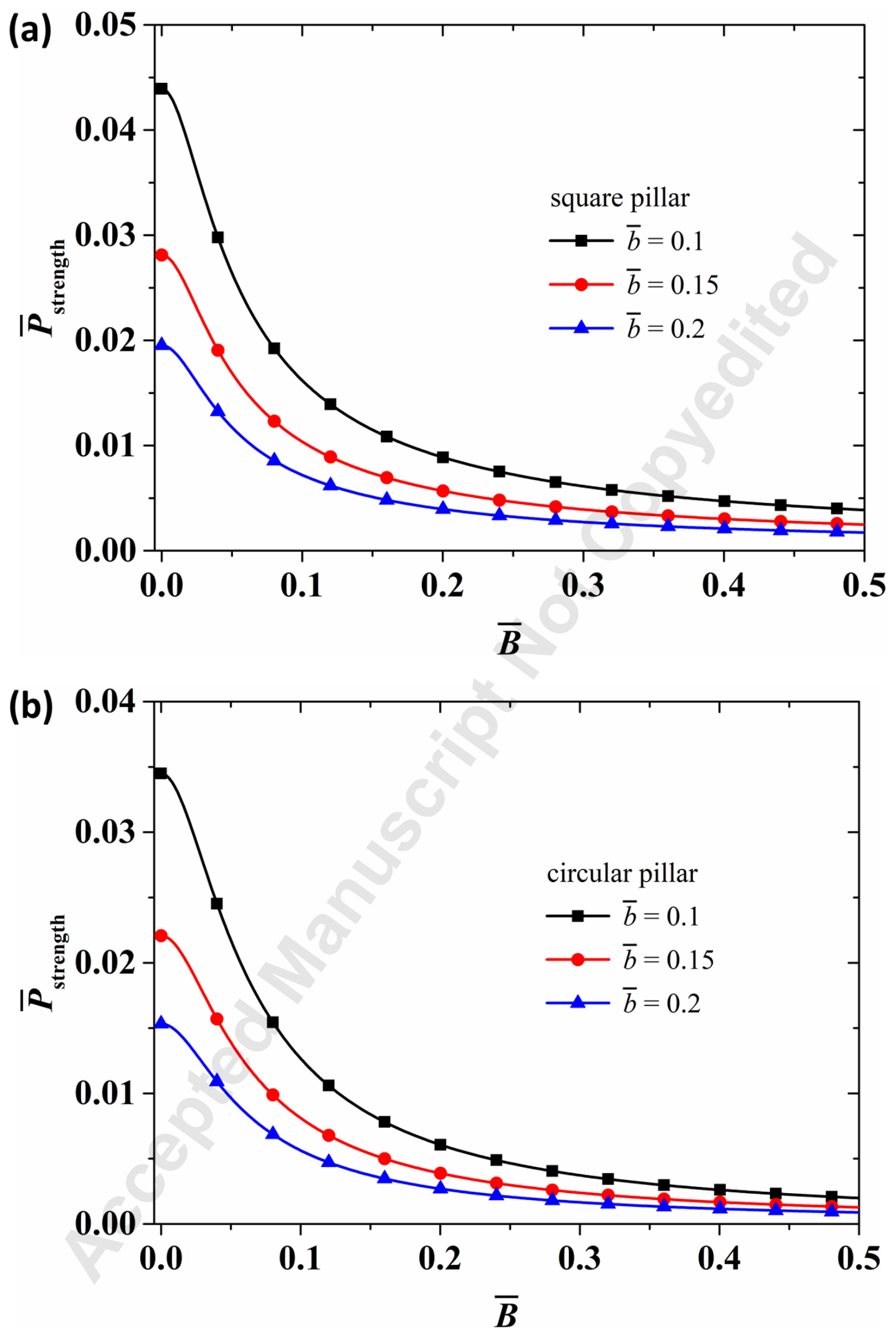

Fig. 8 

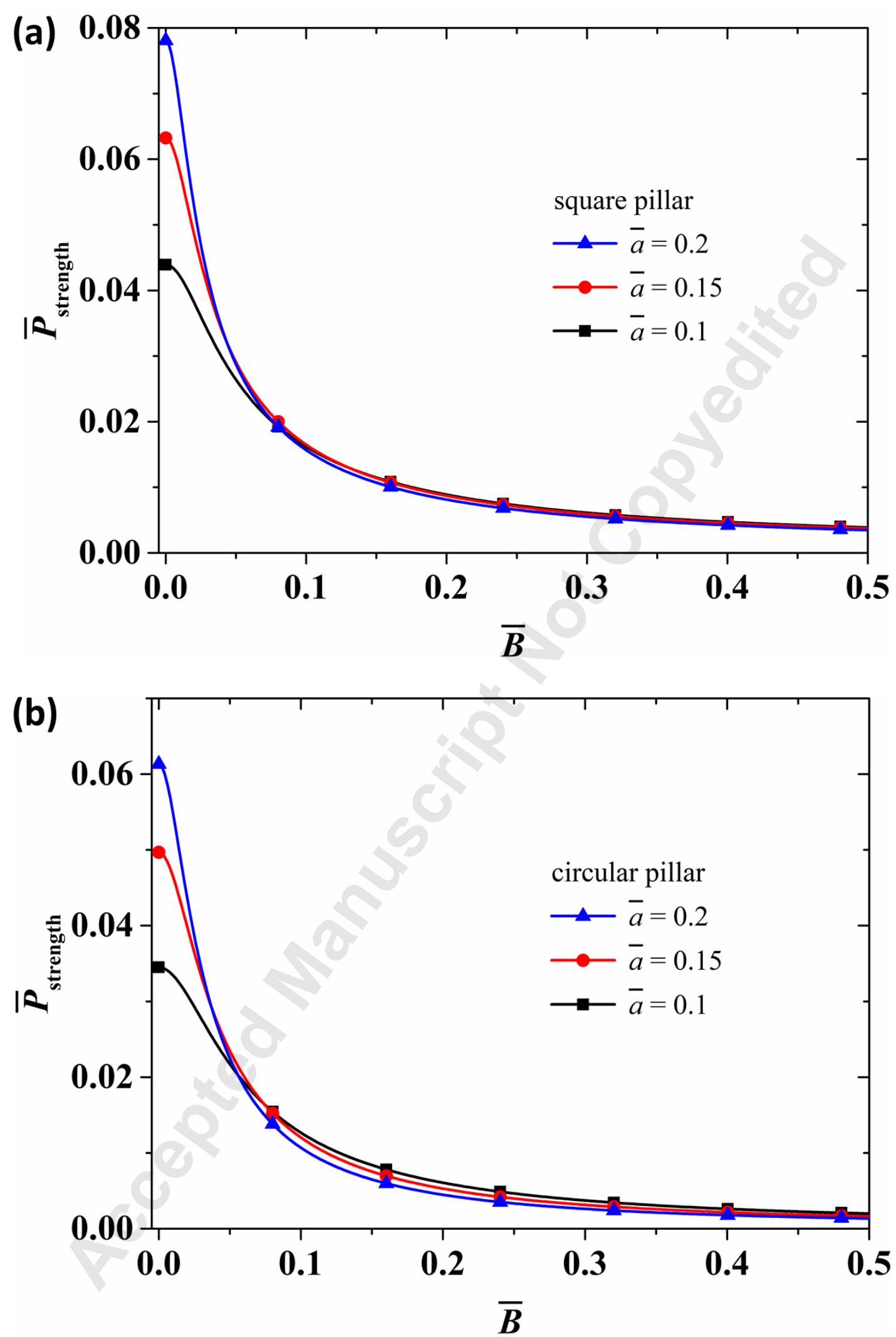

Fig. 9 


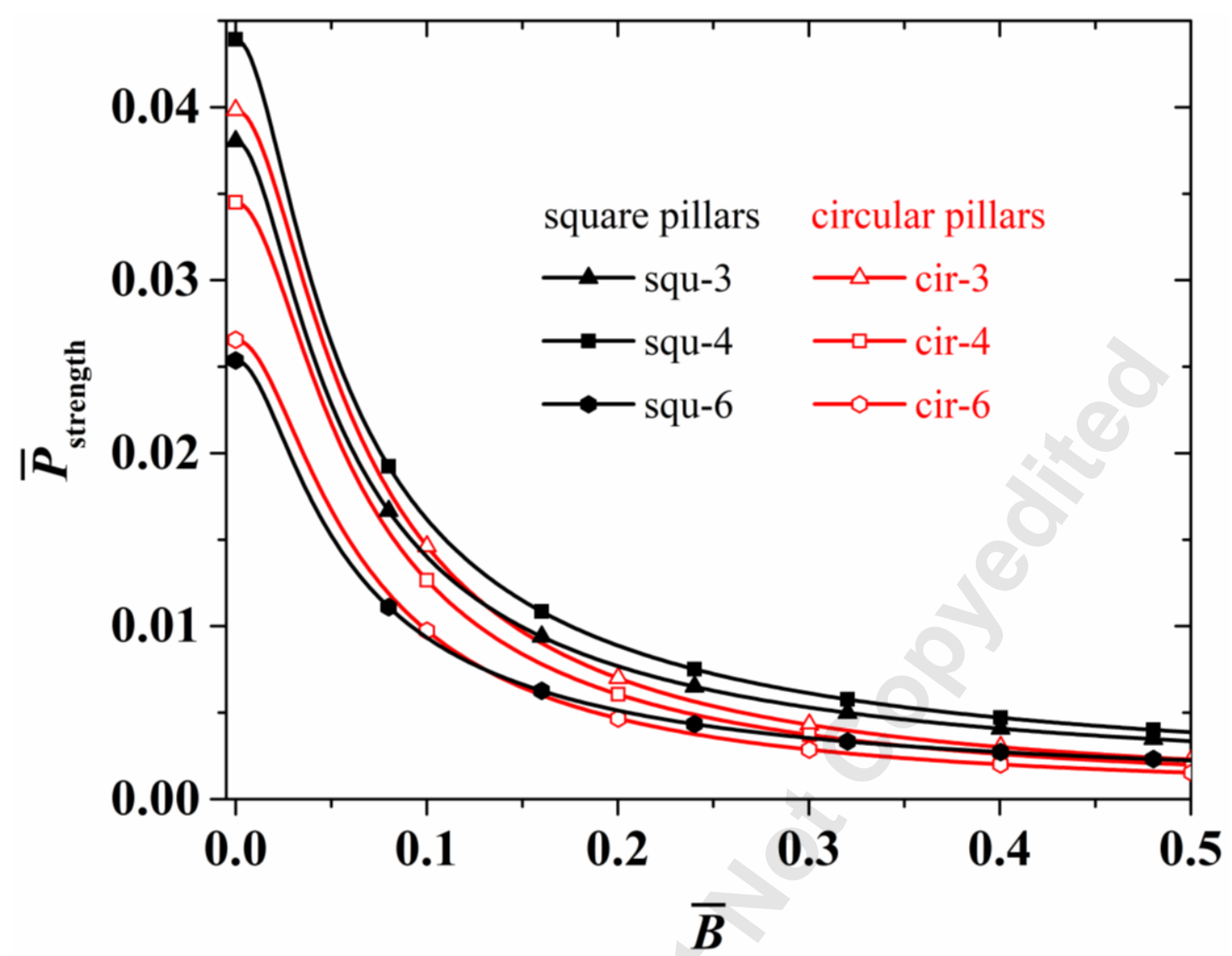

Fig. 10 


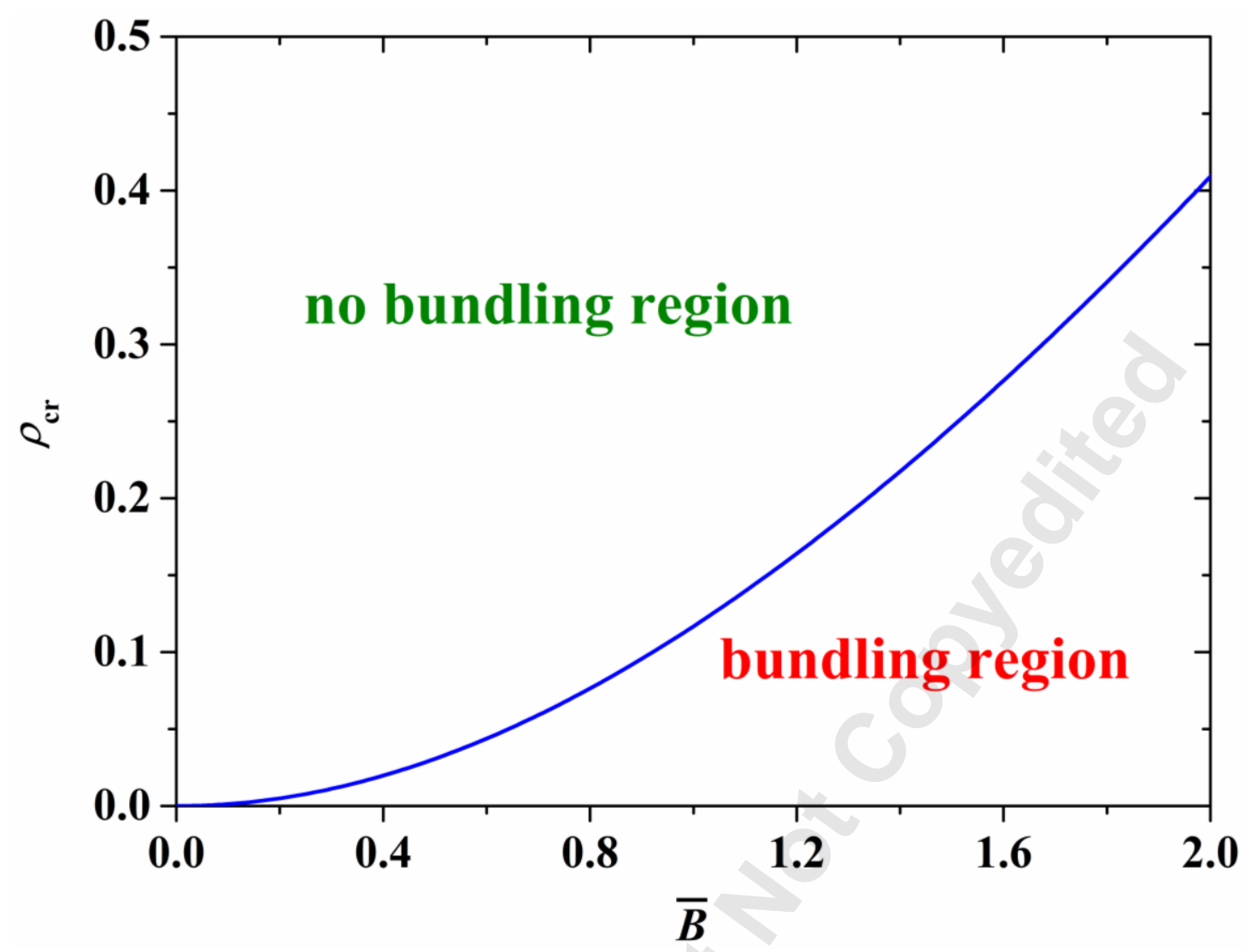

Fig. 11 


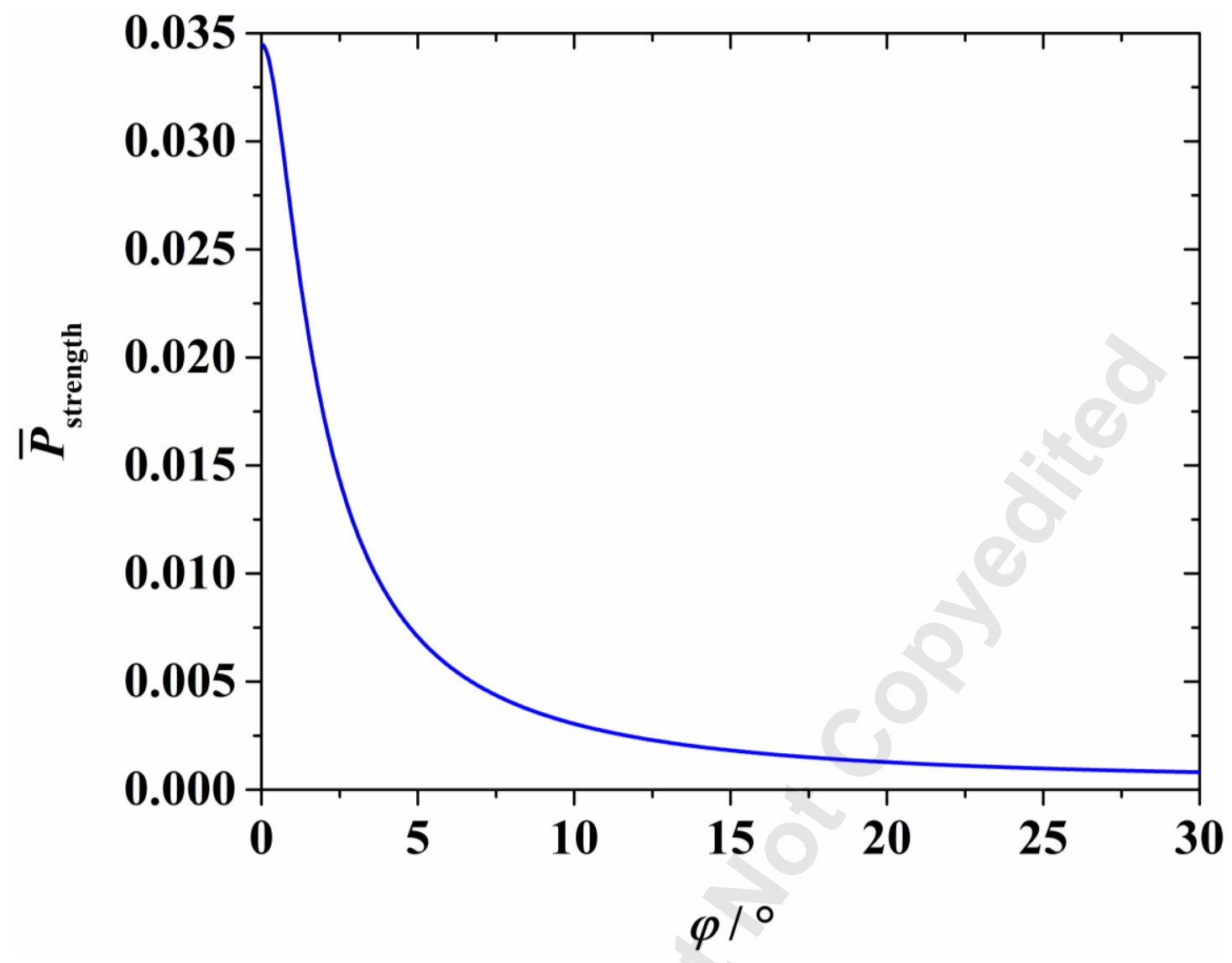

Fig. 12 